\title{
Dynamics of the Delta and Omicron variants of SARS-CoV-2 in the United States: the battle of supremacy in the presence of vaccination, mask usage and antiviral treatment
}

\author{
Calistus N. Ngonghala ${ }^{1,2, *}$, Hemaho B. Taboe ${ }^{3}$, and Abba B. Gumel ${ }^{4,5}$ \\ ${ }^{1}$ Department of Mathematics, University of Florida, Gainesville, FL 32611, USA. \\ 2 Emerging Pathogens Institute, University of Florida, Gainesville, FL 32610, USA. \\ ${ }^{3}$ Laboratoire de Biomathématiques et d'Estimations Forestières, University of Abomey-Calavi, Cotonou, Bénin. \\ ${ }^{4}$ School of Mathematical and Statistical Sciences, Arizona State University, Tempe, Arizona, 85287, USA. \\ ${ }^{5}$ Department of Mathematics and Applied Mathematics, University of Pretoria, Pretoria 0002, South Africa.
}

\begin{abstract}
Multiple safe and effective vaccines and antiviral drugs have been approved or authorized for use against the COVID-19 pandemic in the United States. The effectiveness of these and other intervention measures is threatened by the emergence of numerous SARS-CoV-2 variants of concern. We present a model for studying the transmission dynamics of two of these variants (Delta and Omicron) in the presence of vaccination, treatment of individuals with clinical symptoms of the disease and the use of face masks in the community. The model was fitted using daily case data for the COVID-19 pandemic in the United States corresponding to the period starting from when Omicron first emerged (end of November 2021) to date. It is shown, based on simulating the model with the current COVID-19 data, that the reproduction number of the Delta variant (denoted by $\mathbb{R}_{v d}$ ) is much smaller than one $\left(\mathbb{R}_{v d}=0.28\right.$ ), while that of Omicron (denoted by $\mathbb{R}_{v o}$ ) is approximately equal to unity $\left(\mathbb{R}_{v o}=0.96\right)$. This shows that the Delta variant has essentially died out, and that Omicron is currently the predominant variant of concern in the United States. Furthermore, if the current baseline levels of the control measures being implemented in the United States are maintained, the Omicron variant will also be on a rapid decline (towards elimination). The analysis and simulations of the calibrated model show that vaccine-derived immunity can be achieved in the United States if at least $68 \%$ of the population is fully-vaccinated with either the Pfizer or Moderna vaccine. It is shown that the COVID-19 pandemic can be eliminated in the United States by June of 2022 if the current baseline level of the proportion of individuals that is fully-vaccinated is increased by about $20 \%$. The threshold vaccination coverage needed to achieve the vaccine-derived herd immunity decreases if the vaccination program is combined with a face mask use strategy, particularly one that emphasizes the use of moderate to high quality masks (e.g., surgical or N95 masks). Greater reduction in disease burden (in comparison to the baseline scenario) are recorded if the very high quality N95 masks are prioritized in the community, followed by the moderately-effective surgical masks and then the lowly-effective cloth masks. We also showed that having high percentage of the populace wearing the moderately-effective surgical mask is more beneficial to the community than having low percentage of the populace wearing the highly-effective N95 masks (this result does not hold for the case when cloth masks compliance is compared with that of N95 masks). However, if a certain (fixed) percentage is to give up masking, our study showed that it is more beneficial if they give up wearing surgical masks and not N95 masks (in other words, in a head-to-head comparison, N95 is always superior than surgical mask). This study showed that waning natural and vaccine-derived immunity (if considered individually) offer marginal impact on disease burden, except for the case when they wane at a much faster rate (e.g., within three months), in comparison to the baseline (estimated to be within 9 months to a year). Greater reduction or increase in disease burden is recorded if both the vaccine-derived and natural immunity wane at the same time (rather than the case when we considered only one of them varying, while the other is at baseline). For instance, if both vaccine-derived and natural immunity wane within three months, a $14 \%$ increase in the peak daily cases will be recorded, in comparison to the baseline. For this case, where immunity wanes within three months, our study predicts another (but milder) Omicron wave in the United States that peaks around July 2022 (with the peak 72\% lower than the original Omicron peak). Under this (fast waning) scenario, our study suggests that a fourth dose of the two mRNA vaccines would need to be approved in the United States to aid and accelerate the prospect of SARS-CoV-2 elimination in 2022. It is shown that while the treatment of symptomatic
\end{abstract}

\footnotetext{
*Corresponding author: Calistus N. Ngonghala, email: calistusnn@ ufl.edu
} 
individuals has marginal effect in reducing daily cases of SARS-CoV-2, in comparison to the baseline, it has significant impact in reducing daily hospitalizations. It is further shown that, while treatment significantly reduces hospitalization, the prospects of COVID-19 elimination in the United States is more significantly enhanced if investments in control resources are focused on mask usage and vaccination rather than on treatment.

Keywords: COVID-19; model; vaccination; antiviral treatment, mask; reproduction number; waning immunity; vaccine-derived herd immunity.

\section{Introduction}

The 2019 novel coronavirus (COVID-19), caused by SARS-CoV-2, resulted in an unprecedented pandemic never before seen since the 1918 influenza pandemic [1-5]. As of February 22, 2022, the SARS-CoV-2 pandemic had caused over 427.8 million confirmed cases and 5.9 million deaths globally [6, 7]. The rapid development and deployment of effective vaccines has played a major role in minimizing and mitigating the burden of the pandemic in regions with moderate and high vaccination coverage [8-10]. Specifically, as on February 11, 2022, 27 vaccines have been approved for use in numerous countries around the world [11]. Three of these vaccines, developed by Pfizer/BioNTech, Moderna and Johnson \& Johnson, have been approved by the United States Food and Drugs Administration (FDA) $[12,13]$. The Pfizer and Moderna vaccines are designed by introducing messenger ribonucleic acid (mRNA) that encodes the spike protein of SARS-CoV-2 to elicit an adaptive immune response against the disease [14]. The vaccines, which are primarily administered in a two-dose regimens three to four weeks apart, have an estimated protective efficacy against symptomatic COVID-19 infection of about 95\% [15-17]. A third (booster) dose was approved for these vaccines in November 19, 2021 for all adults from 18 years old [18]. The Johnson and Johnson vaccine, on the other hand, was developed based on using adenovirus vector encoding the SARS-CoV-2 spike protein, and is administered in a single dose regimen. Its efficacy against moderate to severe infection is estimated to be $67 \%$ [19]. We focus on two of the three vaccines being used in the United States.

Despite the deployment of the three safe and effective vaccines in the United States (since late 2020 to early 2021), SARS-CoV-2 transmission in the United States is still on the rise (see, for example, Figure 1). Specifically, as of the time of writing (mid-February 2022), the United States is recording a 7-days average of 172, 951 new COVID-19 cases per day [6]. This is largely due to vaccine hesitancy or refusal (only about $64 \%$ of the population in the United States is fully-vaccinated; with $43 \%$ of this population having received the booster dose as of February 14, 2022) and the emergence of new SARS-CoV-2 variants [20-25]. Thus, the approved vaccines, despite their high efficacy against the original SARS-CoV-2 strain, are currently insufficient to halt the COVID-19 pandemic in the United States. Hence, other control measures, such as antivirals that reduce the risk of disease progression, are needed to add to the armoury of measures for effectively combating or eliminating the COVID-19 pandemic in the United States. It is worth stating that the phenomenon of vaccine hesitancy or refusal during outbreaks of highly contagious and/or fatal vaccine-preventable infectious diseases of humans has a long history, dating back to the 1800s [26-30]. That is, vaccine hesitancy, refusal or general controversy surrounding vaccination programs in humans did not just start with the COVID-19 pandemic.

Two antiviral drugs, namely, Paxlovid (developed by Pfizer Inc.) and Molnupiravir (developed by Merck Inc.), received FDA-Emergency Use Authorization in December 2021, for healthcare providers to use to treat mild-tomoderate COVID-19 in adults and pediatric patients (12 years of age and older weighing at least $40 \mathrm{~kg}$ ) [31]. These antivirals primarily work by altering the genetic code of SARS-CoV-2 and inhibiting it from replicating. Paxlovid, which is administered as three tablets taken together orally twice daily for five days, is estimated to reduce the risk of hospitalization or death by $90 \%$ [32]. Molnupiravir, which is administered as four 200 milligram capsules taken orally every 12 hours for five days, is effective in reducing hospitalization or death by $31 \%$ [33]. Full efficacy of these antivirals is achieved if used during the first five days of onset of symptoms [33, 34].

The unprecedented level of community transmission of SARS-CoV-2 in the United States has resulted in the emer- 
gence of numerous mutants (variants of concern) in the population [35-40]. Of note, the Delta variant (B.1.617.2), which was first identified in India in late 2020 [41], emerged in the United States in July 27, 2021. This variant, which was believed to be more than twice as contagious as previous SARS-CoV-2 variants, quickly spread across the United States causing unprecedented level of hospitalizations and deaths. It remained the most dominant variant (accounting for $99 \%$ of all new cases) until mid- December 2021 (see the region between the dashed green and purple vertical lines in Figure 1), when a new variant (Omicron; B.1.1.529) emerged [37]. Omicron, believed to be three times more contagious than Delta, quickly displaced Delta, and became the predominant variant to date $[17,42,43]$ (see regions to the right of the dashed purple vertical line in Figure 1).
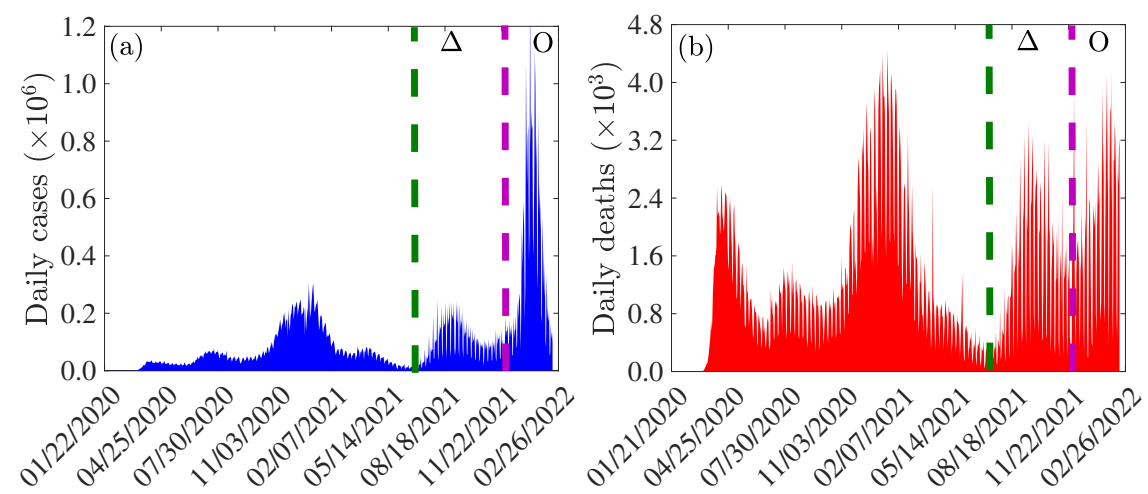

Figure 1: Data for the new COVID-19 daily (a) cases and (b) mortality for the United States for the period of the COVID-19 pandemic from January 2020 until February 2022. Data obtained from the Johns Hopkins University COVID-19 Dashboard [7, 44]. The burden of the Delta variant is illustrated in the regions between the green and purple dashed vertical lines (denoted by $\Delta$ ), while that of the Omicron variant is shown in the regions to the right of the dashed purple vertical line (denoted by $O$ ).

The three FDA-approved vaccines currently being used in the United States were designed against the original SARS-Cov-2 strain, and only offer cross-protective efficacy against the variants [14, 17, 45]. It is, therefore, instructive to theoretically assess, through mathematical modeling and analysis, the population-level effectiveness of the vaccines against the two remaining dominant variants (namely, Delta and the current predominant, Omicron). The current study is based on the design of a mathematical model for assessing the qualitative dynamics of the two dominant SARS-CoV-2 variants co-circulating in the United States (Delta and Omicron). The resulting two-strain model, which takes the form of a deterministic system of nonlinear differential equations, also incorporates the treatment of individuals with clinical symptoms of SARS-CoV-2 (using the two FDA-EUA antivirals discussed above). The model is formulated in Section 2, and fitted using current case data for COVID-19 in the United States (for the period when Omicron first appeared) in Section 2.1. Basic theoretical results, for the existence and asymptotic stability of the disease-free equilibrium of the model, are provided in Section 3. Further, the condition, in parameter space, for achieving vaccine-derived herd immunity is derived. Numerical simulations are presented in Section 4 and a discussion of the results and concluding remarks are presented in Section 5.

\section{Formulation of Mathematical Model}

The model to be developed is for the transmission dynamics of the COVID-19 pandemic in the United States for the scenario where the dominant variant of concern was initially Delta and the new prevailing and currently dominant variant, Omicron, is introduced (this was the scenario in the United States when Omicron emerged in the Fall of 2021). Let $N_{j}(t)$, with $j=\{d, o\}$, represents the variants Delta $(d)$ and Omicron $(o)$, denotes the total population of the United States population at time $t$. This population is stratified into the mutually-exclusive compartments for unvaccinated susceptible $(S(t))$, fully-vaccinated but not boosted susceptible $\left(V_{f}(t)\right.$; these are individuals that have either received the two doses of the FDA-approved Pfizer or Moderna vaccine or the single dose of Johnson \& Johnson vaccine), fully-vaccinated and boosted susceptible $\left(V_{b}(t)\right)$, exposed/latent $\left(E_{j}(t)\right)$, presymptomatic 
$\left(P_{j}(t)\right)$, detected infected individuals from the exposed, presymptomatic, and asymptomatic classes $\left(Q_{j}(t)\right)$, symptomatic infectious within the first five days of onset of symptoms $\left(I_{j 1}(t)\right)$, symptomatic individuals after the first five days of onset of symptoms $\left(I_{j 2}(t)\right)$, asymptomatic infectious or symptomatic infectious with very mild symptoms $\left(A_{j}(t)\right)$, hospitalized $\left(H_{j}(t)\right)$ and recovered $\left(R_{j}(t)\right)$ individuals, so that:

$$
N_{j}(t)=S(t)+V_{f}(t)+V_{b}(t)+E_{j}(t)+P_{j}(t)+Q_{j}(t)+I_{j 1}(t)+I_{j 2}(t)+A_{j}(t)+H_{j}(t)+R_{j}(t) ; j=\{d, o\} .
$$

The population of unvaccinated (wholly) susceptible individuals is increased by recruitment into the population at a rate $\Lambda$ and by the waning of vaccine-derived protective immunity in fully-vaccinated but not boosted (at a rate $\omega_{v f}$ ) and fully-vaccinated and boosted (at a rate $\omega_{v b}$ ) individuals. It is further increased by the loss of natural immunity in individuals who recovered from Delta (at a rate $\omega_{d r}$ ) and Omicron (at a rate $\omega_{o r}$ ) infection. Unvaccinated susceptible individuals acquire infection with the Delta variant at a rate $\lambda_{d}$ and with Omicron at a rate $\lambda_{o}$, where:

$$
\lambda_{d}=\frac{\beta_{d p} P_{d}+\beta_{d a} A_{d}+\beta_{d q} Q_{d}+\beta_{d 1} I_{d 1}+\beta_{d 2} I_{d 2}+\beta_{d h} H_{d}}{N},
$$

and,

$$
\lambda_{o}=\frac{\beta_{o p} P_{o}+\beta_{o a} A_{o}+\beta_{o q} Q_{o}+\beta_{o 1} I_{o 1}+\beta_{o 2} I_{o 2}+\beta_{o h} H_{o}}{N} .
$$

In (2.1)-(2.2), $\beta_{j p}, \beta_{j a}, \beta_{j q}, \beta_{j i}$ and $\beta_{j h}$ (with $j=\{d, o\}$ ) represent, respectively, the transmission rate by presymptomatic, asymptomatic, symptomatic and hospitalized infectious individuals. Unvaccinated susceptible individuals are fully-vaccinated (but not boosted) at a rate $\xi_{v f}$. Individuals in all epidemiological compartments suffer natural death at a rate $\mu$.

Fully-vaccinated (but not boosted) individuals acquire breakthrough infection with Delta (at a rate $\lambda_{d f}$ ) or Omicron (at a rate $\lambda_{o f}$ ), where:

$$
\lambda_{d f}=\left(1-\varepsilon_{d f}\right) \frac{\beta_{d p} P_{d}+\beta_{d a} A_{d}+\beta_{d q} Q_{d}+\beta_{d 1} I_{d 1}+\beta_{d 2} I_{d 2}+\beta_{d h} H_{d}}{N},
$$

and,

$$
\lambda_{o f}=\left(1-\varepsilon_{o f}\right) \frac{\beta_{o p} P_{o}+\beta_{o a} A_{o}+\beta_{o q} Q_{o}+\beta_{o 1} I_{o 1}+\beta_{o 2} I_{o 2}+\beta_{o h} H_{o}}{N},
$$

with $0<\varepsilon_{j f}<1$ representing the cross-protective efficacy the vaccine offers against Delta $\left(\varepsilon_{d f}\right)$ or Omicron $\left(\varepsilon_{o f}\right)$ variant. Fully-vaccinated individuals receive the booster dose (at a rate rate $\xi_{v b}$ ) and lose their vaccine-derived immunity (at a rate $\omega_{v f}$ ). Vaccinated individuals who received the booster dose acquire infection with Delta (at a rate $\lambda_{d b}$ ) or Omicron (at a rate $\lambda_{o b}$ ), where:

$$
\begin{aligned}
& \lambda_{d b}=\left(1-\varepsilon_{d b}\right) \frac{\beta_{d p} P_{d}+\beta_{d a} A_{d}+\beta_{d q} Q_{d}+\beta_{d 1} I_{d 1}+\beta_{d 2} I_{d 2}+\beta_{d h} H_{d}}{N}, \\
& \lambda_{o b}=\left(1-\varepsilon_{o b}\right) \frac{\beta_{o p} P_{o}+\beta_{o a} A_{o}+\beta_{o q} Q_{o}+\beta_{o 1} I_{o 1}+\beta_{o 2} I_{o 2}+\beta_{o h} H_{o}}{N},
\end{aligned}
$$

with $0<\varepsilon_{d b}<1$ and $0<\varepsilon_{o b}<1$ representing the cross-protective efficacy the vaccine offers boosted individuals against Delta and Omicron, respectively.

Exposed (or latent) individuals progress to the pre-symptomatic class at a rate $\sigma_{j e}$. Furthermore, individuals in the pre-symptomatic class progress, at the end of the incubation period, to either the symptomatic class (at a rate $r_{j} \sigma_{j p}$; where $0<r<l$ is the proportion of these individuals that display clinical symptoms of SARS-CoV-2 at the end of the incubation period) or the asymptomatic infectious class (at a rate $(1-r) \sigma_{j p}$ ). Asymptomatic infectious 
individuals recover (naturally) at a rate $\gamma_{j a}$. Infected individuals in the exposed, presymptomatic and asymptomatic classes are detected at rate $\rho_{j}$, detected cases become symptomatic at rate $\psi_{j}$, recover naturally at rate $\gamma_{j q}$, or die naturally at rate $\mu$. Symptomatic individuals during the first five days of onset of symptoms are treated at a rate $\tau_{j 1}$ and progress to the second infectious class at a rate $\alpha_{j 1}$. These individuals are hospitalized at a rate $\phi_{j 1}$ and suffer disease-induced death at a rate $\delta_{j 1}$. Symptomatic individuals that survived the first five days of onset of symptoms are treated at a rate $\tau_{j 2}$, hospitalized at a rate $\phi_{j 2}$, recover at a rate $\gamma_{j 2}$ and die of the disease at a rate $\delta_{j 2}$. Hospitalized individuals are treated at a rate $\tau_{j h}$ and succumb to the disease at a rate $\delta_{j h}$. Recovered individuals lose the infection-acquired (natural) immunity, and revert to the wholly-susceptible class, at a rate $\omega_{j r}$.

The equations for the transmission dynamics of the two variants of concern (Delta and Omicron) in the United States, in the presence of vaccination with any of the FDA- approved vaccines and treatment of symptomatic individuals, is given by the following deterministic system of nonlinear differential equations (the flow diagram of the model is given in Figure 2 and the state variables and parameters of the model are described in Tables S1 and $\mathrm{S} 2$ of the supplementary material, respectively):

$$
\begin{aligned}
\dot{S} & =\Lambda+\omega_{v f} V_{f}+\omega_{v b} V_{b}+\omega_{d r} R_{d}+\omega_{o r} R_{0}-\left(\lambda_{d}+\lambda_{o}+\xi_{v f}+\mu\right) S \\
\dot{V}_{f} & =\xi_{v f} S-\left(\lambda_{d f}+\lambda_{o f}+\xi_{v b}+\omega_{v f}+\mu\right) V_{f} \\
\dot{V}_{b} & =\xi_{v b} V_{f}-\left(\lambda_{d b}+\lambda_{o b}+\omega_{v b}+\mu\right) V_{b} \\
\dot{E}_{j} & =\lambda_{j} S+\lambda_{j f} V_{f}+\lambda_{j b} V_{b}-\left(\sigma_{j e}+\rho_{j}+\mu\right) E_{j} \\
\dot{P}_{j} & =\sigma_{j e} E_{j}-\left(\sigma_{j p}+\rho_{j}+\mu\right) P_{j} \\
\dot{A}_{j} & =\left(1-r_{j}\right) \sigma_{j p} P_{j}-\left(\gamma_{j a}+\rho_{j}+\mu\right) A_{j} \\
\dot{Q}_{j} & =\rho_{j}\left(E_{j}+P_{j}+A_{j}\right)-\left(\gamma_{j q}+\psi_{j}+\mu\right) Q_{j} \\
\dot{I}_{j 1} & =r_{j} \sigma_{j p} P_{j}+\psi_{j} Q_{j}-\left(\tau_{j 1}+\alpha_{j 1}+\phi_{j 1}+\mu+\delta_{j 1}\right) I_{j 1} \\
\dot{I}_{j 2} & =\alpha_{j 1} I_{j 1}-\left(\tau_{j 2}+\gamma_{j 2}+\phi_{j 2}+\mu+\delta_{j 2}\right) I_{j 2} \\
\dot{H}_{j} & =\phi_{j 1} I_{j 1}+\phi_{j 2} I_{j 2}-\left(\tau_{j h}+\gamma_{j h}+\mu+\delta_{j h}\right) H_{j} \\
\dot{R}_{j} & =\gamma_{j a} A_{j}+\gamma_{j q} Q_{j}+\tau_{j 1} I_{j 1}+\left(\tau_{j 2}+\gamma_{j 2}\right) I_{j 2}+\left(\tau_{j h}+\gamma_{j h}\right) H_{j} \\
& -\left(\omega_{j r}+\mu\right) R_{j}, \text { with } j \in\{d, o\}
\end{aligned}
$$

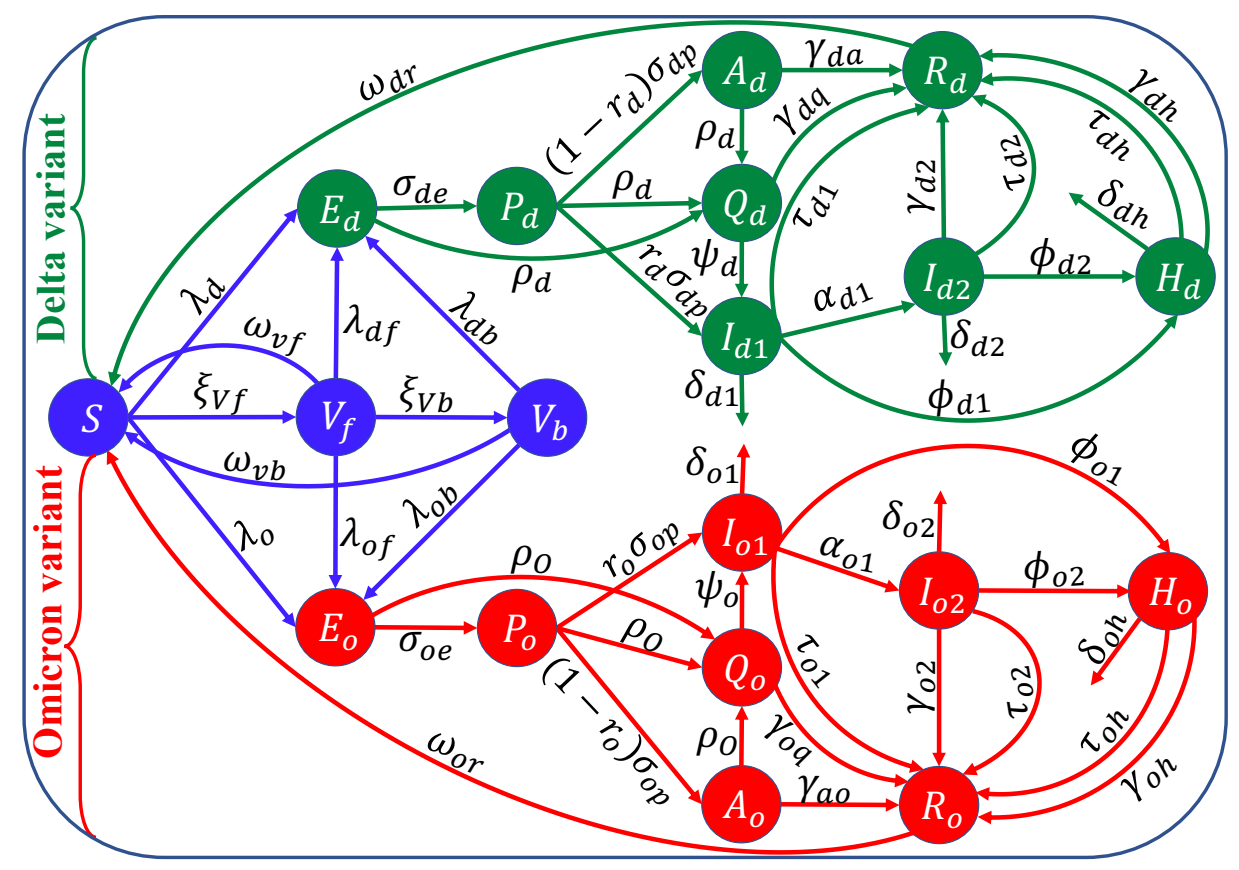

Figure 2: Flow diagram of the model (2.7). Although recruitment into the population and natural deaths occur (at the rate $\Lambda$ and $\mu$, respectively), these rates are not illustrated in the flow diagram to make it less crowded and easier to follow. The state variables and parameters are described in Tables S1 and S2 of the supplementary material. 
medRxiv preprint doi: https://doi.org/10.1101/2022.02.23.22271394; this version posted February 24, 2022. The copyright holder for this preprint

(which was not certified by peer review) is the author/funder, who has granted medRxiv a license to display the preprint in perpetuity.

It is made available under a CC-BY-NC-ND 4.0 International license.

Some of the main assumptions made in the formulation of the model (2.7) are

(a) Only the Delta and Omicron variants are co-circulating (i.e., they are the most predominant of all the variants of concern at the current time). Specifically, we consider the scenario where Delta was the dominant strain and then Omicron emerged (as was the case in the United States during the fall of 2021).

(b) A well-mixed population, where every individual is equally likely to mix with every other individual in the community. We also consider an endemic scenario, to account for demographic (birth and natural death) processes. This is needed to account for the vaccination of children (five years of age and older).

(c) The vaccines only offer cross-protective efficacy against the variants (since all the current FDA-approved vaccines were developed for the original/resident strain that was circulating in the United States during the early stages of the pandemic).

(d) Natural immunity and vaccine-derived immunity for fully-vaccinated and boosted humans wane over time.

The model (2.7) is an extended version of numerous COVID-19 transmission models in the literature that consider the dynamics of multiple co-circulating variants, such as those in [46-55] by, inter alia, including:

1. Incorporating a booster dose (this was not considered in [46-55]).

2. Incorporating the treatment of symptomatic individuals during and after the first five days of onset of symptoms (treatment against COVID-19 was not considered in [46-54]). It is worth mentioning that although the agent-based model considered in Matrajt et al. [55] was used to evaluate the impact of treatment on COVID19 dynamics, the model does not explicitly account for waning of immunity and the use of a booster shot against the disease.

3. Allowing for waning vaccine-derived and natural immunity over time (this was not considered in [46-55].

4. Explicitly including the impact of voluntary testing to detect infected individuals who do not have clinical symptoms of the disease (this was not done in [46-55] ). It should be stated that detection of infected individuals who do not display clinical symptoms of the disease (i.e., those in the exposed/latent class, $E$, presymptomatic class, $P$, and asymptomatic class, $A$ ) is crucial in allowing the model to fit the daily confirmed case data reasonably well (we would not get a good model fitting, with respect to the daily confirmed case data) if this feature is not included in the model). In particular, including detection of infected individuals in these classes that do not exhibit disease symptoms makes it possible to be able to fit confirmed daily cases in the model to confirmed daily cases from the available data.

\subsection{Model Fitting and Parameter Estimation}

The model (2.7) has several parameters, some of which are known (see Table S3) and some of which are unknown. The key unknown parameters that will be estimated are the effective community transmission rates for infectious individuals in the classes $p_{d}, A_{d}, Q_{d}, I_{d 1}, I_{d 2}$, and $H_{d}$ (i.e., $\beta_{d p}, \beta_{d a}, \beta_{d q}, \beta_{d 1}, \beta_{d 2}$, and $\beta_{d h}$ ), the rate at which individuals are fully-vaccinated $\left(\xi_{v f}\right)$, and the rate at which fully-vaccinated individuals are boosted $\left(\xi_{v b}\right)$. These parameters will be estimated by fitting the model (2.7) to confirmed daily COVID-19 case data for the U.S. for the period from November 28, 2021 to January 31, 2022. The estimation involves computing the best set of parameter values that minimizes the sum of the square difference between the observed new daily confirmed case data and the new daily cases from the model (2.7) (i.e., $\left.\rho_{d}\left(E_{d}+P_{d}+A_{d}\right)+r_{d} \sigma_{d p} P_{d}+\rho_{o}\left(E_{o}+P_{o}+A_{o}\right)+r_{o} \sigma_{o p} P_{o}\right)$ through a nonlinear regression procedure. The $95 \%$ confidence intervals for the estimated parameters are determined using a bootstrapping technique [56-58]. It should be mentioned that fitting the model to daily confirmed case data is useful in mitigating mistakes arising when cumulative is fitted to deterministic models [59]. The estimated model parameters and the corresponding confidence intervals are presented in Table S4.

The results obtained for the fitting of the daily new COVID-19 cases in the United States, for the period when 
Omicron first emerged (November 28, 2021) until January 31, 2022 (i.e., the region to the left of the dashed vertical green line), is depicted in Figure 3 (a). This figure shows a very good fit between the model output (blue curve) and the observed data (red dots). Furthermore, we show in this figure the prediction of the model for the daily COVID-19 cases for approximately a seven-week period after January 31, 2022 (i.e., for the Period from February 1, 2022 to March 18, 2022), as illustrated by the segment of the graph to the right of the dashed vertical black line of Figure 3 (a). This segment of the Figure 3 (a) clearly shows that the model (2.7) predicts the observed data for the period from February 1-22, 2022 perfectly (solid green curve). The model was then simulated (using the fixed and fitted parameter baseline values in Tables S3 and S4) and compared with the observed cumulative mortality data. The results obtained, depicted in Figure 3 (b), show a very good fit. Further, this figure also shows perfect model prediction for the period from February 1, 2022 to March 18, 2022 (see region to the right of the dashed vertical black line).
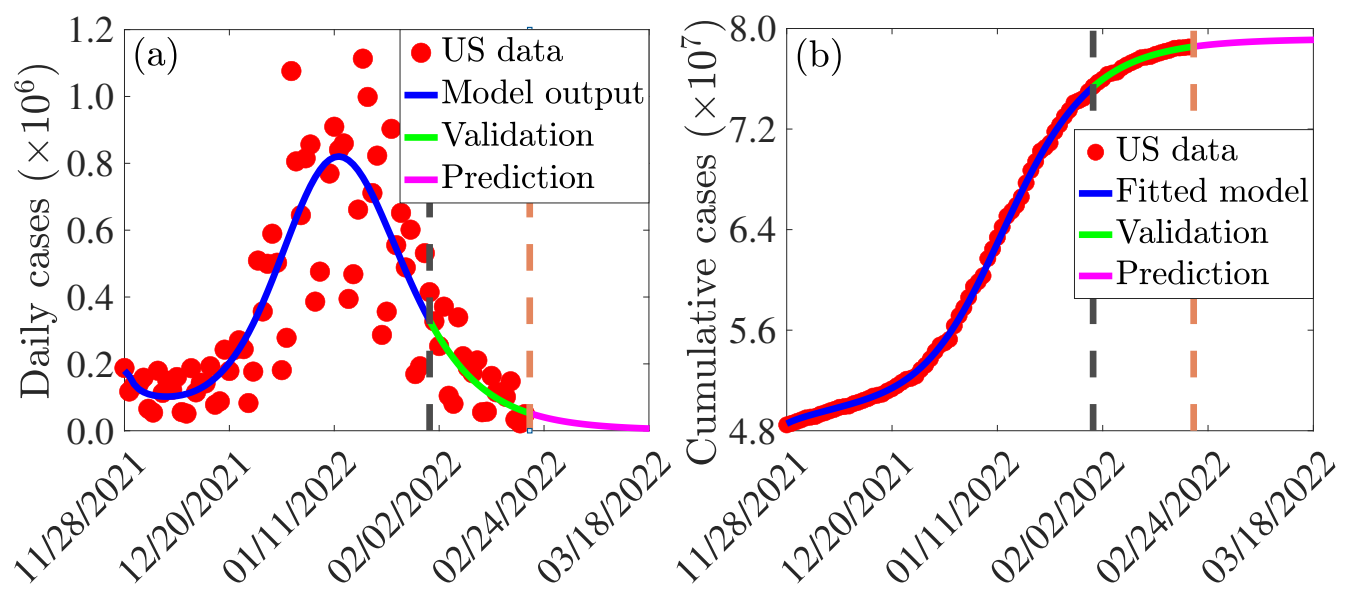

Figure 3: (a) Time series illustration of the least squares fit of the model (2.7), showing the model's output for the daily cases (blue curve) compared to the observed daily confirmed cases for the United States (red dots) from November 28, 2021 to January 31, 2022 (segment to the left of the dashed vertical black line). (b) Simulation result of the (2.7), showing cumulative COVID-19 cases for the United States as a function of time, using the fixed and estimated baseline parameter values given in Tables S3 and S4. The segment from February 1, 2022 to March 18, 2022 (i.e., solid green and magenta curves or the entire segment to the right of the dashed black vertical line) illustrates the performance of the model (2.7) in predicting the daily and cumulative cases in the United States.

\section{Theoretical Results}

In this section, the basic qualitative properties of the model (2.7), with respect to the disease-free equilibrium, will be explored.

\subsection{Disease-free equilibrium}

The disease-free equilibrium of the model (2.7) is given by:

$$
\begin{aligned}
& \left(S^{*}, V_{f}^{*}, V_{b}^{*}, E_{d}^{*}, P_{d}^{*}, A_{d}^{*}, Q_{d}^{*}, I_{d 1}^{*}, I_{d 2}^{*}, H_{d}^{*}, R_{d}^{*}, E_{o}^{*}, P_{o}^{*}, A_{o}^{*}, Q_{o}^{*}, I_{o 1}^{*}, I_{o 2}^{*}, H_{o}^{*}, R_{o}^{*}\right) \\
& =\left(S^{*}, V_{f}^{*}, V_{b}^{*}, 0,0,0,0,0,0,0,0,0,0,0,0,0,0,0,0\right)
\end{aligned}
$$

where,

$$
S^{*}=\frac{\Lambda\left(\omega_{v b}+\mu\right)\left(\xi_{v b}+\omega_{v f}+\mu\right)}{\left[\left(\xi_{v b}+\xi_{v f}+\omega_{v f}+\mu\right)\left(\omega_{v b}+\mu\right)+\xi_{v b} \xi_{v f}\right] \mu}, V_{f}^{*}=\frac{\xi_{v f}}{\xi_{v b}+\omega_{v f}+\mu} S^{*}, V_{b}^{*}=\frac{\xi_{v b} \xi_{v f}}{\left(\omega_{v b}+\mu\right)\left(\xi_{v b}+\omega_{v f}+\mu\right)} S^{*} .
$$


The vaccination reproduction number of the model with respect to variant $j$ (with $j, j \in\{d, o\}$ ), denoted by $\mathbb{R}_{j v}$, can be obtained using the next generation operator method [60,61]. It is given by the following expression:

$$
\mathbb{R}_{j v}=\mathbb{R}_{j p}+\mathbb{R}_{j a}+\mathbb{R}_{j q}+\mathbb{R}_{j 1}+\mathbb{R}_{j 2}+\mathbb{R}_{j h}
$$

where,

$$
\begin{aligned}
\mathbb{R}_{j p} & =\frac{\beta_{j p} \sigma_{j e} G_{j 1} G_{j 2} G_{j a} G_{j q} G_{j h}}{G_{j e} G_{j p} G_{j a} G_{j q} G_{j 1} G_{j 2} G_{j h}} H^{*}, \quad \mathbb{R}_{j a}=\frac{\beta_{j a} \sigma_{j e} \sigma_{j p} G_{j q} G_{j 1} G_{j 2} G_{j h}\left(1-r_{j}\right)}{G_{j e} G_{j p} G_{j a} G_{j q} G_{j 1} G_{j 2} G_{j h}} H^{*}, \\
\mathbb{R}_{j q} & =\frac{\beta_{j q} G_{j 1} G_{j 2} G_{j h} \rho_{j}\left\{\left[G_{j a}+\left(1-r_{j}\right) \sigma_{j p}\right] \sigma_{j e}+G_{j a} G_{j p}\right\}}{G_{j e} G_{j p} G_{j a} G_{j q} G_{j 1} G_{j 2} G_{j h}} H^{*}, \\
\mathbb{R}_{j 1} & =\frac{\beta_{j 1} G_{j 2} G_{j h}\left\{\left\{\left[G_{j a}+\left(1-r_{j}\right) \sigma_{j p}\right] \psi_{j} \rho_{j}+r_{j} \sigma_{j p} G_{j a} G_{j q}\right\} \sigma_{j e}+\psi_{j} \rho_{j} G_{j a} G_{j p}\right\}}{G_{j e} G_{j p} G_{j a} G_{j q} G_{j 1} G_{j 2} G_{j h}} H^{*} \\
\mathbb{R}_{j 2} & =\frac{\beta_{j 2} G_{j h} \alpha_{j 1}\left\{\left\{\left[G_{j a}+\left(1-r_{j}\right) \sigma_{j p}\right] \psi_{j} \rho_{j}+r_{j} \sigma_{j p} G_{j a} G_{j q}\right\} \sigma_{j e}+\psi_{j} \rho_{j} G_{j a} G_{j p}\right\}}{G_{j e} G_{j p} G_{j a} G_{j q} G_{j 1} G_{j 2} G_{j h}} H^{*}, \\
\mathbb{R}_{j h} & =\frac{\beta_{j h}\left(\phi_{j 1} G_{j 2}+\phi_{j 2} \alpha_{j 1}\right)\left\{\left\{\left[G_{j a}+\left(1-r_{j}\right) \sigma_{j p}\right] \psi_{j} \rho_{j}+r_{j} \sigma_{j p} G_{j a} G_{j q}\right\} \sigma_{j e}+\psi_{j} \rho_{j} G_{j a} G_{j p}\right\}}{G_{j e} G_{j p} G_{j a} G_{j q} G_{j 1} G_{j 2} G_{j h}} H^{*},
\end{aligned}
$$

with,

$$
H^{*}=\frac{\left[S^{*}+\left(1-\varepsilon_{j f}\right) V_{f}^{*}+\left(1-\varepsilon_{j b}\right) V_{b}^{*}\right]}{S^{*}+V_{f}^{*}+V_{b}^{*}} .
$$

In (3.2), $G_{j e}=\sigma_{j e}+\rho_{j}+\mu, G_{j p}=\sigma_{j p}+\rho_{j}+\mu, G_{j a}=\gamma_{j a}+\rho_{j}+\mu, G_{j q}=\gamma_{j q}+\psi_{j}+\mu ; G_{j 1}=\tau_{j 1}+\alpha_{j 1}+$ $\phi_{j 1}+\mu+\delta_{j 1}, G_{j 2}=\tau_{j 2}+\gamma_{j 2}+\phi_{j 2}+\mu+\delta_{j 2}$ and $G_{j h}=\gamma_{j h}+\tau_{j h}+\mu+\delta_{j h}$. It is convenient to define:

$$
\mathbb{R}_{c}=\max \left\{\mathbb{R}_{d v}, \mathbb{R}_{o v}\right\} .
$$

The quantity $\mathbb{R}_{c}$ is the vaccination reproduction number of the model (2.7). It measures the average number of new SARS-CoV-2 cases generated by a typical infectious individual introduced in a community where a certain proportion of the susceptible individuals is fully-vaccinated. The result below follows from Theorem 2 of [61].

Theorem 3.1. The disease-free equilibrium of the model (2.7) is locally-asymptotically stable if $\mathbb{R}_{c}<1$, and unstable if $\mathbb{R}_{c}>1$.

The epidemiological implication of Theorem 3.1 is that a small influx of individuals infected with the SARS-CoV-2 virus will not generate a large outbreak in the community. Thus, the disease can be effectively controlled (if the initial number of infected individuals is small enough) if the reproduction number $\left(\mathbb{R}_{c}\right)$ can be brought to (and maintained) a value less than one.

Using the baseline values of the fixed and estimated parameters of the model (2.7), given in Tables S3 and S4 (used to generate the fittings in Figure 3), shows that the constituent reproduction numbers for the Delta $\left(\mathbb{R}_{d v}\right)$ and Omicron $\left(\mathbb{R}_{o v}\right.$ ) are $\mathbb{R}_{d v}=0.2782<1$ (with $95 \%$ confidence interval $\mathbb{R}_{d v} \in(0.1991,0.5197)$ ) and $\mathbb{R}_{o v}=0.9602$ (with $95 \%$ confidence interval, $\mathbb{R}_{o v} \in(0.6206,1.7509)$, so that the overall vaccination reproduction number of the $\operatorname{model}\left(\mathbb{R}_{c}\right)=\max \left\{\mathbb{R}_{d v}, \mathbb{R}_{o v}\right\}=\mathbb{R}_{o v}=0.9602$. This suggests that, based on the data for the COVID-19 dynamics in the United States for the period November 28, 2021 to January 31, 2022, the Delta variant has essentially died out (owing to its very low reproduction number, less than one) and Omicron has displaced Delta and become the predominant variant in the United States. Furthermore, since the reproduction number of Omicron (and, hence, of the model (2.7) itself) is less than one (albeit slightly), it follows (from Theorem 3.1 or, equivalently, Theorem 3.2 below) that the Omicron variant may also be dying out if the current baseline levels of control measures in the United States are maintained. In other words, our analysis (and data fitting, using the most updated data) suggests that the COVID-19 pandemic in the United States may be entering its declining phase, and that elimination is indeed feasible if current baseline levels of control measures are maintained. 


\subsection{Vaccine-derived herd immunity threshold}

To obtain an expression for the vaccine-derived herd immunity threshold [54,62, 63], it is instructive to recall that the basic reproduction number for variant $j$, denoted by $\mathbb{R}_{0 j}$, with $j \in\{d, o\}$, is obtained from (3.1) by setting $V_{f}=V_{b}=\varepsilon_{j f}=\varepsilon_{j b}=0$. It follows that $\mathbb{R}_{0 j}=\left.\mathbb{R}_{c}\right|_{V_{f}=V_{b}=\varepsilon_{j f}=\varepsilon_{j b}=0}$. Let $f_{v}=\min \left\{f_{v f}, f_{v b}\right\}$ represents the proportion of individuals who are fully-vaccinated only $\left(f_{v f}\right)$ and those that are fully-vaccinated and boosted $\left(f_{v b}\right)$ at steady-state. Furthermore, let $\varepsilon_{j v}=\min \left\{\varepsilon_{j f}, \varepsilon_{j b}\right\}$ represents the minimum vaccine efficacy for fully-vaccinated $\left(\varepsilon_{j f}\right)$ and boosted $\left(\varepsilon_{j b}\right)$ individuals. Setting $\mathbb{R}_{c}=1$ in (3.3), and solving for $f_{v}$, gives the following expression for the vaccine-derived herd immunity threshold for the model (2.7):

$$
f_{v}=\frac{1}{\varepsilon_{j v}}\left(1-\frac{1}{\mathbb{R}_{0 j}}\right)=f_{v}^{c} .
$$

It follows from (3.4) that $\mathbb{R}_{c}<(>) 1$ if $f_{v}^{c}>(<) 1$. Thus, vaccine-induced herd immunity can be achieved in the community (and the disease be eliminated) if $f_{v}>f_{v}^{c}$. Theorem 3.1 can be written in terms of the herd immunity threshold, as below.

Theorem 3.2. The disease-free equilibrium of the model (2.7) is locally-asymptotically stable if $f_{v}>f_{v}^{c}\left(i . e ., \mathbb{R}_{c}<\right.$ $1)$, and unstable if $f_{v}<f_{v}^{c}\left(\right.$ i.e., $\left.\mathbb{R}_{c}>1\right)$.

Using the baseline parameter values in Tables S3 and S4, and the herd immunity threshold expression (3.4) shows that the value of the vaccine-derived herd immunity threshold for the United States is $f_{v}^{c}=0.68$. Thus, our study shows that vaccine-derived herd immunity can be achieved in the United States (during the current time when both Omicron and Delta are circulating, with the former being the overwhelming predominant variant) if at least $68 \%$ of the population of individuals living in the United States are fully-vaccinated with either the Pfizer and Moderna vaccine. In line with Theorem 3.2, COVID-19 can be eliminated in the United States if this level of vaccination coverage is attained. Data from $[64,65]$ shows that about $64 \%$ of the United States population is fully-vaccinated as of February 14, 2022. Hence, our study shows that the United States can eliminate the pandemic if about $4 \%$ of the proportion of unvaccinated individuals or individuals who have received only one dose of Pfizer or Moderna vaccine become fully-vaccinated with either of these two vaccines.

\section{Numerical Simulations}

The model (2.7) is simulated, using the baseline values of the fixed and fitted parameter values in Tables S3 and S4, to assess the impact of vaccination, boosting of vaccine-derived immunity, testing (and detection of infected individuals with no clinical symptoms of the disease) and the treatment of symptomatic individuals, on the dynamics of COVID-19 in the United States.

Figure 4 depicts contour plots of the control reproduction number of the model, as a function of the efficacy of the two vaccines (Pfizer or Moderna) against acquisition of infection with Delta or Omicron (defined as $\varepsilon_{v}=$ $\min \left\{\varepsilon_{v f}, \varepsilon_{v b}\right\}$ ) and the fraction of the U.S. population fully-vaccinated at steady-state (defined as $f_{v}=\min \left\{f_{v d}, f_{v o}\right\}$ ). For the case where mask usage in the community is maintained at baseline level, this figure shows a decrease in the reproduction number with increasing values of the vaccine efficacy and coverage. Specifically, for the case when the cross-protective vaccine efficacy against the two variants is set at $60 \%$, population-wide vaccine-derived herd immunity (associated with the reduction of the reproduction number to a value below one) can be achieved if $84 \%$ of the U.S. population is fully-vaccinated with either the Pfizer or Moderna vaccine (Figure 4(a)). It follows from this contour plot that the herd immunity requirement reduces to $65 \%$ if the vaccines offer $80 \%$ cross-protective efficacy against the two variants.

Further simulations are carried out for the case where the baseline face mask usage in the community is increased by $10 \%$, for various mask types. Figure 4(b) shows that if surgical masks are prioritized, the herd immunity 
requirement corresponding to $60 \%(80 \%)$ cross-protective vaccine efficacy against the variants now reduces to $80 \%(60 \%)$, in comparison to the $84 \%$ (65\%) coverage recorded for the case with baseline face mask coverage depicted in Figure 4(a). Furthermore, if N95 masks are prioritized, the herd immunity requirement corresponding to the cross-protective vaccine efficacy of $60 \%$ (80\%) further reduces to $77 \%$ (58\%). In summary, the contour plots in Figures 4(b) and (c) show that the proportion of individuals who need to be fully-vaccinated to achieve herd immunity reduces with increasing coverage of face masks in the community (from the baseline face mask usage), and the level of reduction achieved depends on the quality of the mask used (specifically, greater reduction in herd immunity level needed to eliminate the disease is achieved if the high-quality N95 masks are prioritized, in comparison to the scenario where the moderate quality surgical masks are prioritized).

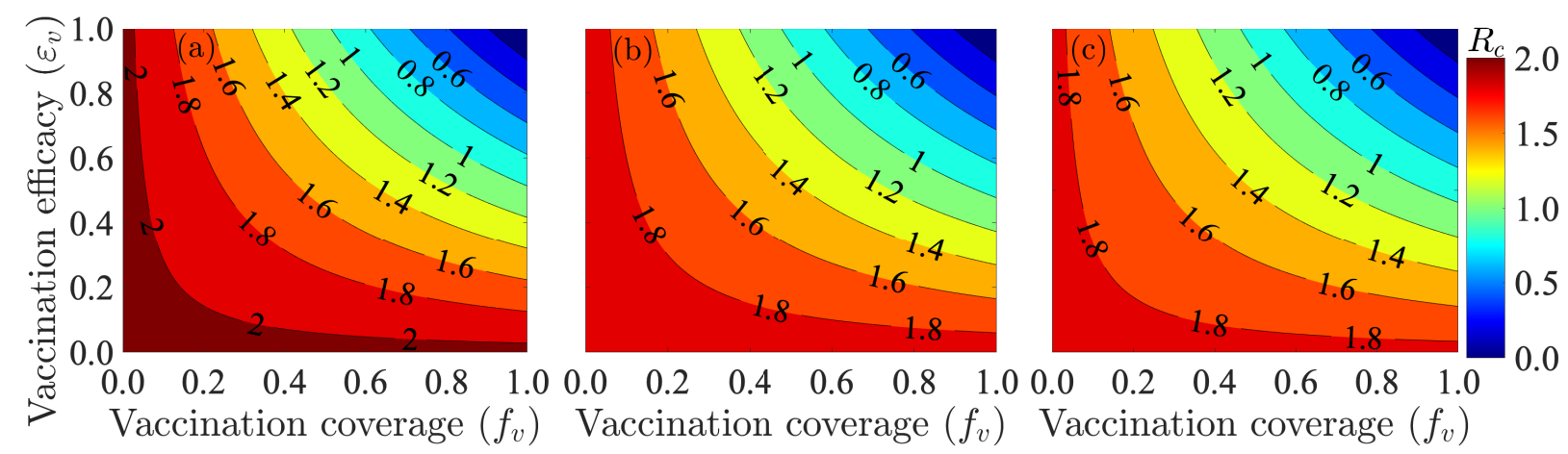

Figure 4: Contour plots of the control reproduction number of the model (2.7), $\mathbb{R}_{c}$, as a function vaccine coverage (i.e., proportion of fully-vaccinated individuals, $f_{v}$ ) and cross-protective vaccine efficacy against the variants $\left(\varepsilon_{v}=\min \left(\varepsilon_{v f}, \varepsilon_{v b}\right)\right)$ for the case when (a) mask coverage is maintained at its baseline value, (b) surgical mask is prioritized and the coverage in its usage is increased by $10 \%$ from its baseline value, (c) N95 mask is prioritized and the coverage in its usage is increased by $10 \%$ from its baseline value. The values of all other parameters used in the simulations are as given by the baseline values in Tables S3 and S4.

\subsection{Assessing the impact of vaccination coverage}

The impact of vaccination coverage (i.e., the rate at which unvaccinated susceptible individuals become fullyvaccinated) is monitored by simulating the model (2.7) with various values of the vaccination rate $\left(\xi_{v f}\right)$. To exclusively monitor the impact of vaccination, the simulations are carried out for the special case of the model with no treatment (i.e., all treatment-related parameters and state variables of the model are set to zero). The simulation results obtained, depicted in Figure 5, show a significant decrease in the daily (Figure 5 (a)) and cumulative (Figure 5 (b)) COVID-19 cases with increasing vaccination coverage of fully-vaccinated individuals (in relation to the baseline value of the vaccination coverage), as expected. For instance, this figure show that increasing the baseline value of the fully-vaccinated rate $\left(\xi_{v f}\right)$ by $20 \%$ resulted in a $12 \%$ reduction in daily cases at the peak (Figure 5 (a), gold curve), in comparison to the baseline scenario (Figure 5 (a), blue curve). Further reduction (at least $23 \%$ at the peak) is achieved if the baseline value of the fully-vaccinated vaccination coverage is increased by $40 \%$ (Figure 5 (a), green curve). On the other hand, if the baseline value of the fully-vaccinated vaccination coverage is decreased, for instance, by $20 \%$, the daily cases at the peak increases (compare magenta curve with blue curve of Figure 5 (a)). Similar reductions in cumulative number of cases are recorded with increasing fully-vaccinated vaccination coverage rate (Figure 5 (b)). In summary, Figure 5 shows that both the daily and cumulative COVID-19 cases can be significantly decreased with even a relatively small increase in the baseline value of the fully-vaccinated vaccination rate (e.g., a $20 \%$ increase from baseline coverage rate of the fully-vaccinated coverage rate). This figure also shows that the COVID-19 pandemic can be eliminated in the United States, under the baseline vaccination coverage scenario, in late July of 2024. The time-to-elimination is accelerated with increasing values of the baseline fully-vaccinated coverage rate. For instance, Figure 5 (a) shows that elimination can be achieved in June of 2022 if the current baseline fully-vaccinated coverage rate is increased by $20 \%$. 

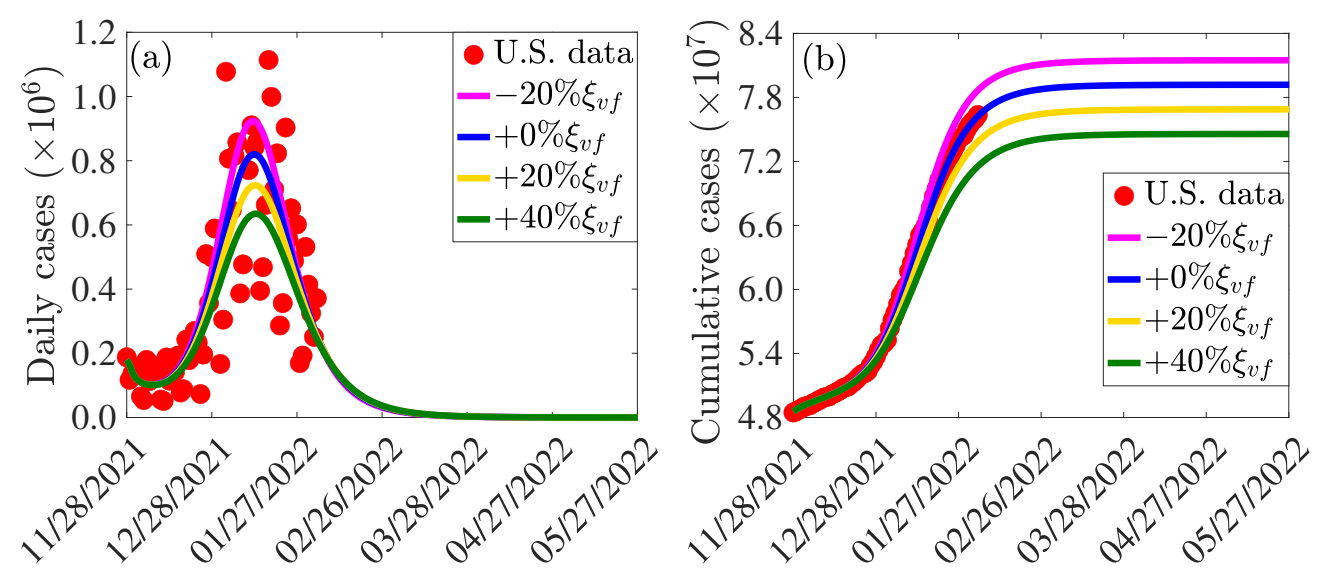

Figure 5: Simulations of the model (2.7) showing the effect of increases or decreases in fully-vaccinated vaccination coverage rate $\left(\xi_{v f}\right)$ on the COVID-19 pandemic in the United States. (a) Daily cases, as a function of time, for various values of the fully-vaccinated vaccination coverage rate. (b) Cumulative cases, as a function of time, for various values of the fully-vaccinated vaccination coverage rate. The values of all other parameters used in these simulation are given by the baseline values in Tables S3 and S4.

\subsection{Assessing the impact of additional increase in mask usage from baseline}

The incremental impact of masking coverage (and type) on the effectiveness of the vaccination program (here, too, no treatment of symptomatic individuals is allowed). The simulations are carried out using baseline values of the parameters in Tables S3 and S4, with various values of mask coverage $\left(c_{m}\right)$ and types (cloth masks, with estimated efficacy of $30 \%$ (i.e., $\varepsilon_{m}=0.3$ ); surgical masks, with estimated efficacy of $70 \%$ (i.e., $\varepsilon_{m}=0.7$ ); and N95 mask, with estimated efficacy of $95 \%$ (i.e., $\varepsilon_{m}=0.95$ ). The results obtained, depicted in Figure 6 , show that, for the fully-vaccinated vaccination coverage (using Pfizer/Moderna vaccine) maintained at the baseline value, increasing coverage of mask usage in the community $\left(c_{m}\right)$ resulted in a dramatic reduction in daily COVID-19 cases, particularly if the moderately-effective surgical or the highly-effective N95 masks are prioritized (Figure 6 (a)). For example, under this scenario (with fully-vaccinated vaccination coverage kept at baseline), a $10 \%$ increase in mask coverage with surgical mask (Figure 6 (a), dashed gold curve) or N95 respirator (Figure 6 (a), dashed green curve) will result in a $26 \%$ and $35 \%$ decrease in peak daily cases,respectively, in comparison to the baseline (Figure 6 (a), blue curve). Further reductions are recorded if the coverages of the two mask types are increased by $20 \%$ (Figure 6 (a), solid gold and solid green curves). For the case where only the ineffective cloth masks are priorities, a $10 \%$ increase in baseline coverage of these masks (Figure 6 (a), dashed magenta curve) will result in approximately $11 \%$ decrease in daily cases at the peak, in comparison to the baseline. A further increase to $20 \%$ coverage from baseline will result in a $22 \%$ decrease in the peak daily cases at baseline (Figure 6 (a), solid magenta curve). Finally, similar reductions in cumulative cases are also recorded with increasing values of the baseline coverage of each of the mask type used in the community (Figure 6 (b)).

It should be noted that, in all of the simulations carried out in this subsection (and with the same increase in mask coverage, $c_{m}$ ), greater reductions are recorded using N95 masks, followed by surgical mask, and then cloth masks (as expected). Furthermore, our results show that a $20 \%$ increase in the baseline value of surgical mask coverage (Figures 6 (a)-(b), solid gold curve) is more effective (in reducing cases) than a $10 \%$ increase in baseline N95 coverage (Figures 6 (a)-(b), dashed green curve). In other words, our simulations show that, having more people wear the moderately-effective surgical mask is more effective than having fewer people wear the highly-effective N95 masks. However, this result, which is consistent with that reported in [66] does not hold when N95 or surgical mask is compared with the low-effective cloth mask. Specifically, a $20 \%$ increase in the baseline coverage of cloth mask (Figures 6 (a)-(b), solid magenta curve) is not more effective than a $10 \%$ increase in the baseline coverage of either surgical (Figures 6 (a)-(b), dashed gold curve) or N95 (Figures 6 (a)-(b), dashed green curve) mask. 
Finally, it should be mentioned that relaxing mask usage from the current baseline (as is currently the case in some jurisdictions in the United States partially or fully relaxing mask mandates [67-71] will result in a re-bounce in disease burden. For example, for the case where mask coverage is decreased by $10 \%$ from the current baseline level, our simulations show that a $4-5 \%$ increase in the peak daily cases will be recorded if either the surgical mask (Figure 6(c), dashed purple curve) or N95 mask (6(c), solid magenta curve) is prioritized. Similar increases in the cumulative COVID-19 cases are recorded with a decrease in baseline coverage of each of the mask type used in the community (Figure $6(\mathrm{~d})$ ). Thus, our simulations show that, based on the current data and baseline levels of COVID-19 interventions implemented in the United States, relaxing mask mandates will result in increase in new cases (up to about $5 \%$ if surgical or N95 are prioritized). Furthermore, giving up masking with surgical mask by a certain proportion is less detrimental than giving up masking with N95 mask by the same proportion (in other words, reducing coverage of surgical mask by $10 \%$, for instance, is less detrimental to the community than reducing the coverage of N95 mask by 10\%). This figure also shows that fewer people giving up N95 masks (e.g., $5 \%$ ) is less detrimental than more people (e.g., 10\%) giving up surgical masks.
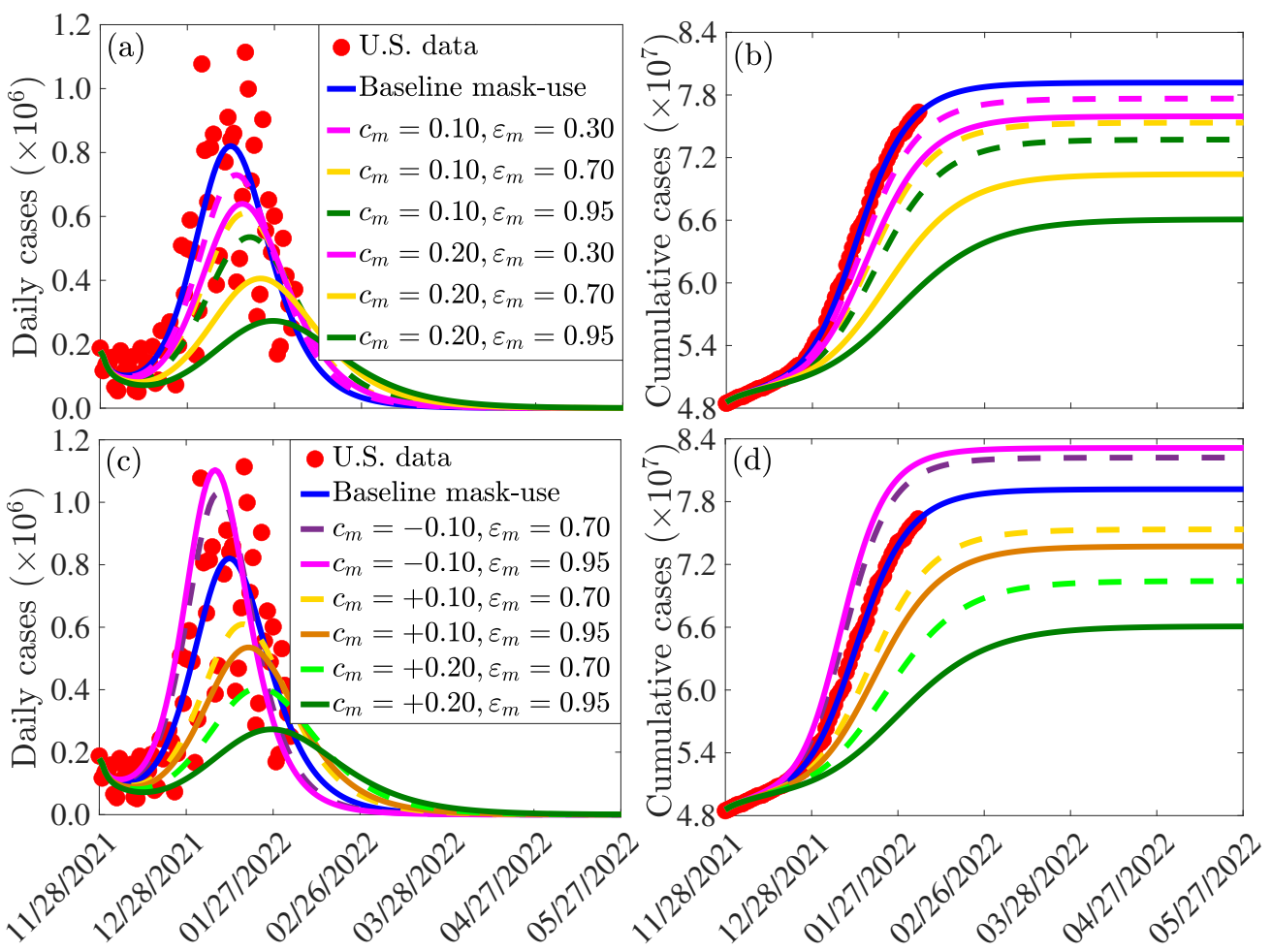

Figure 6: Simulations of the model (2.7) showing the incremental impact of mask coverage $\left(c_{m}\right)$ and mask type (cloth masks, with $\varepsilon_{m}=0.3$; surgical masks, with $\varepsilon_{m}=0.7$; and $\mathrm{N} 95$ respirators, with $\varepsilon_{m}=0.95$ ) on the daily ((a) and (c)) and cumulative ((b) and (d)) COVID-19 cases in the United States, as a function of time. The values of the other parameters used in these simulations are as given in Tables S3 and S4.

In summary, the simulations in this subsection show that, for the case where fully-vaccinate vaccination coverage is maintained at baseline level (and no treatment strategy is implemented), increasing the baseline value of mask coverage reduces the daily and cumulative COVID-19 cases, and the level of reduction increases with increasing quality of the mask type that is prioritized in the community. For communities that prioritize the use of the moderately-effective surgical and the highly-effective N95 masks only (i.e., communities that discourage the use of cloth masks, which are known to be generally ineffective [72-75], having more people (e.g., 20\% increase from baseline level) wear surgical masks is more beneficial to the community than having fewer people (e.g., 10\% increase from baseline) wear N95 masks. In other words, in this context (with only surgical and N95 masks available), investment in significant increase in the coverage of surgical masks (from their baseline level) may be more effective than a small increase in the baseline coverage of N95 mask. 


\subsection{Assessing the combined impact of vaccination and masking up}

The model (2.7) is further simulated to assess the combined impacts of mask coverage $\left(c_{m}\right)$, mask type (cloth masks, with $\varepsilon_{m}=0.3$; surgical masks, with $\varepsilon_{m}=0.7$; and N95 respirators, with $\left.\varepsilon_{m}=0,95\right)$ and fully-vaccinated vaccination coverage rate $\left(\xi_{v f}\right)$ on the daily and cumulative number of COVID-19 cases in the United States. For these simulations, we consider a $20 \%$ increase in the baseline values of both mask coverage $\left(c_{m}\right)$ and fully-vaccinated vaccination coverage rate $\left(\xi_{v f}\right)$. The results obtained, depicted in Figure 7, show that using the ineffective cloth masks will result to a $33 \%$ reduction in the baseline peak daily cases (Figure 7 (a); magenta vs. blue curves). This reduction is better than the $22 \%$ reduction in peak daily cases for the scenario where only the baseline vaccination coverage was increased (Figure 6(a), solid magenta curve). The reduction in the number of cases is more significant if masks of higher quality are prioritized. Specifically, if the moderately-effective surgical masks are prioritized, the simulation show that up to $60 \%$ reduction in peak daily cases can be achieved (Figure 7 (a), gold curve). The reduction increases to $74 \%$ if the highly-effective N95 masks are prioritized (Figure 7 (a), green curve). These reductions exceed the $51 \%$ (67\%) reductions recorded, for the corresponding scenarios, when the fully-vaccinated vaccination coverage was maintained at its (Figure 6 (a), solid gold and green curves). Similar reductions in the cumulative cases are recorded with increasing baseline vaccination and mask coverage for each of the mask types used in the community (Figure 7 (b)).
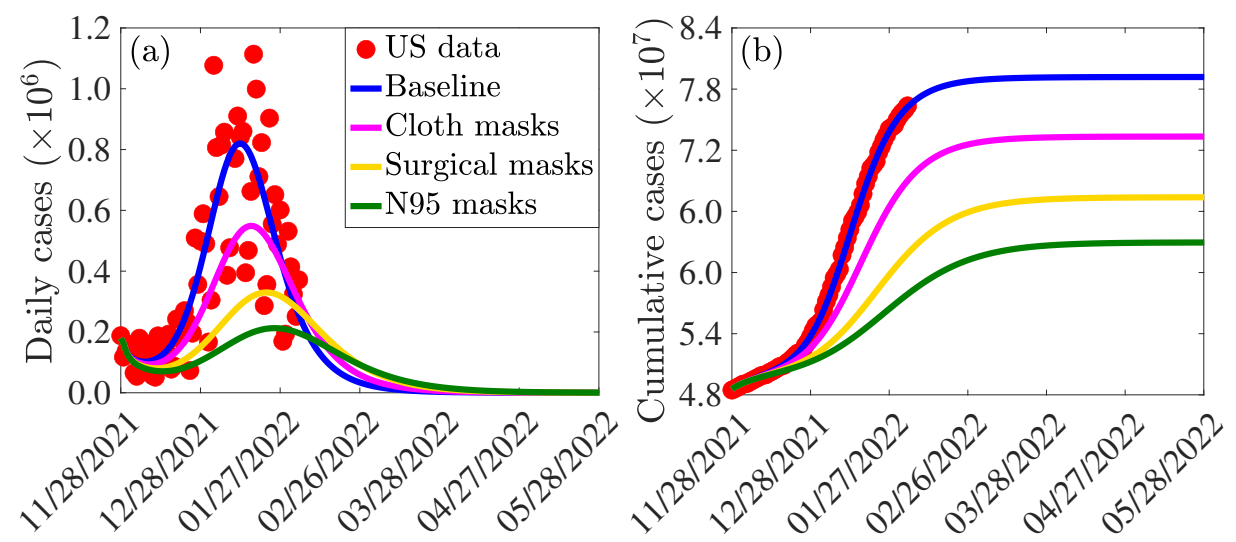

Figure 7: Simulations of the model (2.7), showing the combined incremental impact of mask coverage $\left(c_{m}\right)$, mask type (cloth masks, with $\varepsilon_{m}=0.3$; surgical masks, with $\varepsilon_{m}=0.7$; and N95 respirators, with $\varepsilon_{m}=0,95$ ) and fully-vaccinated vaccination coverage rate $\left(\xi_{v f}\right)$ on the daily ((a)) and cumulative ((b)) COVID-19 cases in the United States, as a function of time. In these simulations, the mask and fully-vaccinated vaccination coverage rates are increased by $20 \%$ from their respective baseline values. The values of the other parameters used in these simulations are as given in Tables S3 and S4.

Contour plots of the control reproduction number $\left(\mathbb{R}_{c}\right)$ of the model (2.7), as a function of the vaccine rate coverage $\left(\xi_{v f}\right)$ and additional mask coverage $\left(c_{m}\right)$ for different mask types, are generated to determine the optimal combinations of the mask and vaccination coverage that can reduce the reproduction number to a value less than unity (so that the pandemic can be eliminated). The results obtained, depicted in Figure S1 in the Supplementary Material, show that, for a community that prioritizes surgical masks (and the coverage in its usage is held at baseline), the reproduction number can be brought down to a value less than unity if up to 1.8 million individuals become fullyvaccinated per day (i.e., if the fully-vaccinated vaccination rate is $\xi_{v f}=0.00532$ per day). If the baseline coverage of the surgical mask is increased by $20 \%$, the reproduction number can be brought to a value less than one if 1.2 million individuals are fully-vaccinated each day (i.e., if $\xi_{v f}=0.00364$ ). However, if surgical mask coverage can be increased by $50 \%$ from its baseline level, only about 490, 000 individuals need to be fully-vaccinated per day to bring the reproduction number to a value less than one. On the other hand, if N95 masks are prioritized, the requirement for high daily vaccination rate decreases. For instance, if the N95 coverage is increased by $20 \%$ from its baseline, only about 1 million individuals need to be fully-vaccinated every day to bring the reproduction number to a value less than one (i.e., we need $\xi_{v f}=0.00308$ per day, under this scenario). If the baseline coverage of N95 
in the community can be increased by $50 \%$, the number of individuals that need to be fully-vaccinated everyday to reduce the reproduction number below one dramatically reduces to 110,880 (i.e., $\xi_{v f}=0.00033$ per day in this case). In summary, the contour plots in Figure S1 show that the prospects of eliminating COVID-19 in the United States is more promising if increases in baseline levels of fully-vaccinated vaccination coverage are combined with increases in baseline levels of face mask coverage that prioritizes moderate (surgical) or high quality (N95 masks).

\subsection{Assessing the impact of waning vaccine-derived and natural immunity}

In this section, the model (2.7) is simulated to assess the effect of waning immunity (both vaccine-derived and natural) on the dynamics of the SARS-CoV-2 pandemic in the United States. We first considered the case where only the waning rate of the vaccine-derived immunity (for both fully-vaccinated and boosted individuals) varies from its baseline value, while the waning rate of natural immunity remains at its baseline value (of nine months). If the vaccine-derived immunity wanes within three months (i.e., $\omega_{v}=\omega_{v f}=\omega_{v b}=1 /(0.25 \times 365)=0.011$ per day), our simulations show that the peak daily cases increases by $8 \%$, in comparison to the case where the vaccinederived immunity wanes at the baseline value of 9 months (Figure 8 (a), magenta curve, in comparison to blue curve). A lower peak of the daily cases is recorded if the vaccine-derived immunity wanes in 6 months (Figure 8 (a), gold curve, in comparison to blue curve). If, on the other hand, it takes 4 years for the vaccine-derived immunity to wane, our simulations show a marginal decrease in the peak daily cases (about $4 \%$ decrease), in comparison to the baseline (Figure 8 (a), green curve, in comparison to blue curve). Similar trends are observed if only waning natural immunity is varied, while the waning rates of vaccine-derived immunity (for both fully-vaccinated and boosted individuals) are maintained at their baseline values (Figure 8 (b)) and when both vaccine-derived and natural immunity are varied from their respective baseline values (Figure 8 (c)). Further, same trends are observed with respect to the cumulative cases for each of the three variable waning rate scenarios (Figures 8 (d)-(f)).
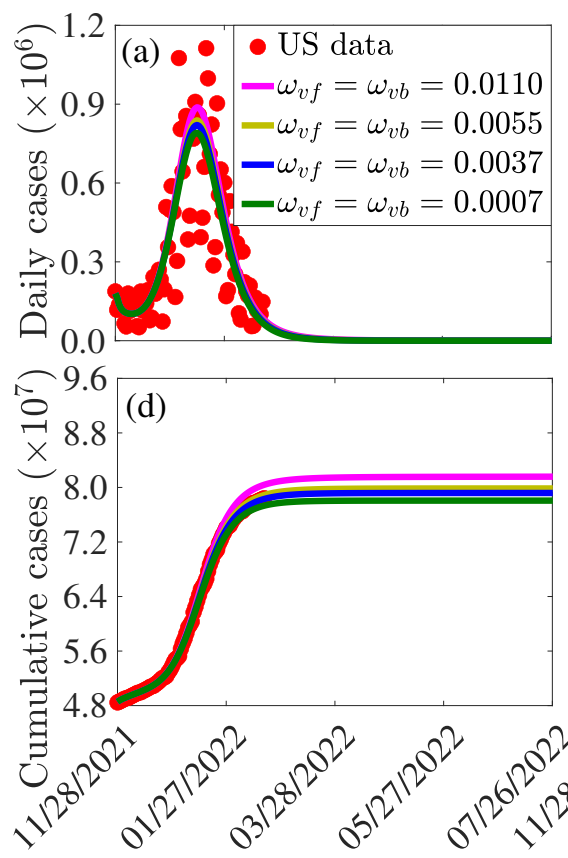
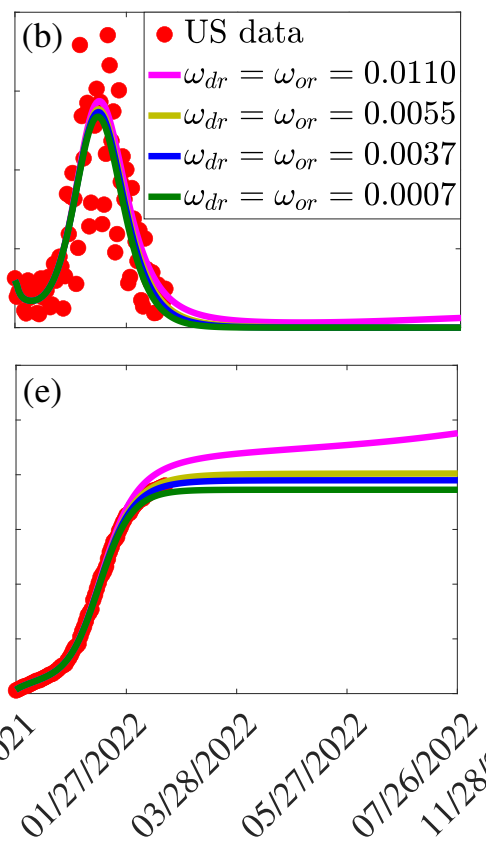
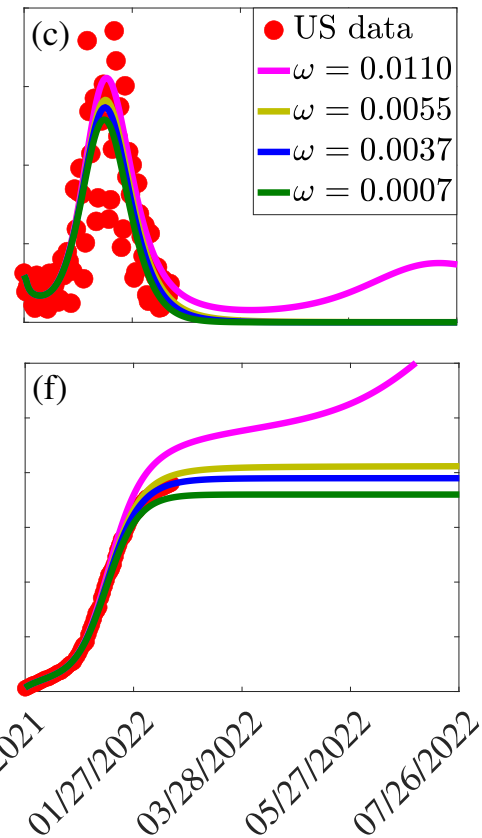

Figure 8: Simulations of the model (2.7), showing (a)-(c) new daily and (d)-(f) cumulative COVID-19 cases in the United States, as a function of time, for various values of the waning rate of (a) and (d) vaccine-derived immunity in fully-vaccinated and boosted individuals $\left(\omega_{v}=\omega_{v f}=\omega_{v b}\right)$, (b) and (e) natural immunity for the Delta and Omicron variants $\left(\omega_{r}=\omega_{d r}=\omega_{o r}\right)$, and (c) and (f) both vaccine-derived and natural immunity $\left(\omega_{v r}=\omega_{v f}=\right.$ $\left.\omega_{v b}=\omega_{d r}=\omega_{o r}\right)$. The durations for the waning of immunity were taken to be 3 months (i.e., $\left.\omega=0.0110\right)$ per day), six months ( $\omega=0.0055$ per day), nine months ( $\omega=0.0037$ per day) and 48 months ( $\omega=0.0007$ per day), respectively.The values of the other parameters used in these simulations are as given in Tables S3 and S4. 
It is worth mentioning that it can be seen from the simulations depicted in Figure 8 that the increase or decrease in disease burden (i.e., daily or cumulative cases) recorded when the waning rates of both the vaccine-derived and natural immunity are varied exceed that for the scenario where only the vaccine-derived or the natural immunity is allowed to vary from baseline. In particular, for the case where both the vaccine-derived and natural immunity wane within 3 months (i.e., $\omega=\omega_{v f}=\omega_{v b}=\omega_{d r}=\omega_{o r}=0.0110$ per day), a $14 \%$ increase in the peak new daily cases is recorded, in comparison to the baseline (we record $8 \%$ increase when only vaccine-derived immunity is allowed to vary, as mentioned above). Furthermore, for this case, our simulations suggest that another wave of the pandemic that peaks by mid July 2022 (with peak new daily cases about $72 \%$ lower than those for the baseline scenario) is predicted to occur (Figure 8 (c), magenta curve). It should, however, be stressed that the simulations in Figure 8 suggest that decreasing waning rate of the vaccine-derived immunity (up to about 4 years) seems to have only a marginal impact in decreasing COVID-19 cases. This may be due to the fact that the two vaccines (Pfizer/Moderna) are highly efficacious against the original SARS-CoV-2 strain, and also moderately-efficacious in the level of cross-protection they offer against the Delta and Omicron variants.

\subsection{Assessing the impact of antiviral treatment against COVID-19}

In this section, the model (2.7) is simulated to assess the potential impact of the two antiviral drugs (Paxlovid and Molnupiravir) that received FDA Emergency Use Authorization for use in the United States to treat individuals with clinical symptoms of COVID-19. As of the time of writing (February 2022), the two antivirals are not widely deployed in the United States. Consequently, there isn't real data to realistically estimate the treatment rates. It should be recalled that in the model (2.7), treatment is offered to symptomatic individuals (during the first five days or after the first five days of onset of symptoms) and hospitalized individuals at rates $\tau_{j k}$ (where $j=\{d, o\}$, representing the two variants; and $k=1,2$, representing the two symptomatic compartments $I_{j 1}$ and $I_{j 2}$ ). In the absence of the aforementioned data, our simulations will be carried out for the special case where the treatment rate to be the same for each treated compartment (i.e., $\tau_{j 1}=\tau_{j 2}=\tau_{j h}=\tau$, with $j=\{d, o\}$ ).

The simulation results obtained are depicted in Figure 9. This figure shows, first of all, that treatment seems to only have marginal impact in reducing daily new cases (Figure 9 (a)). For instance, if $\tau=0.8$ (i.e., if symptomatic individuals are offered treatment with any of the two antivirals within $1 / 0.8=1.25$ days on average), the reduction in daily cases, in comparison to the baseline case is marginal (compare blue and green curves in Figure 9(a)). On the other hand, our simulations show that treatment has a more significant impact on reducing daily hospitalization (Figure 9 (b)). For instance, if it takes an average five days to treat a symptomatic individual (i.e., $\tau=0.2$ ), a $37 \%$ reduction in the peak hospitalization will be recorded, in comparison to the baseline (Figure 9(b), compare magenta and blue curves). Under this scenario, and with all other intervention-related parameters kept at their baseline, it will take until June 30, 2024 before zero COVID-related hospitalizations can be achieved in the United States.

If the treatment rate is increased to $\tau=0.4$ (i.e., if it takes an average of 2.5 days to treat a symptomatic individual), the reduction in baseline peak hospitalization increases to 55\% (Figure 9(b), compare gold and blue curves). In this case, zero hospitalization can be achieved by June 19, 2024. Finally, the reduction in baseline peak hospitalization increases to $72 \%$ if $\tau=0.8$ (Figure 9(b), compare green and blue curves). For this scenario, zero hospitalization can be achieved by June 6, 2024 (this will be visible if Figure 9 is extended to 2024). In summary, the simulations in this subsection show that while treatment only has marginal impact on reducing number of cases, it does have significant impact in reducing daily hospitalizations. With all other interventions (vaccination and masking) maintained at their baseline values, our simulations show that treatment, even at perhaps the highest possible rate (such as a rate associated with treating symptomatic individuals within a day or two of onset of symptoms) will delay COVID-19 elimination (as measured, in these simulations, in terms of attaining zero hospitalization) until 2024. Such elimination can be achieved by increasing the effectiveness and coverages of vaccination and/or mask usage. In other words, the simulations in this section suggest that, while treatment reduces hospitalization (a highly desirable goal too), the prospect of COVID-19 elimination is enhanced by focusing investments on mask usage and vaccination than on treatment. 

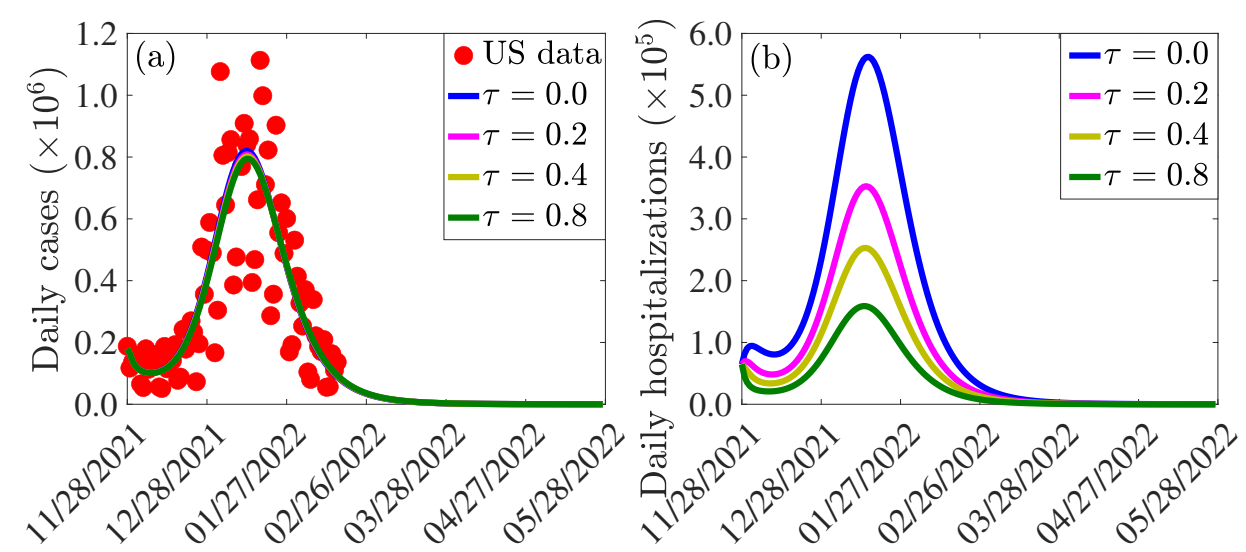

Figure 9: Simulations of the model (2.7) depicting the impact of treatment of symptomatic infectious and hospitalized individuals infectious on the (a) confirmed daily COVID-19 cases, and (c) daily COVID-19 hospitalizations in the United States. The treatment rate $(\tau)$ is given by $\tau=\tau_{j 1}=\tau_{j 2}=\tau_{j h}, j \in\{d, o\}$. The other parameter values used for the simulations are presented in Tables S3 and S4.

\section{Discussion and conclusion}

One of the main success stories in the effort to control the spread of the devastating novel 2019 coronavirus pandemic (COVID-19, caused by SARS-CoV-2) was the rapid development and deployment of safe and effective vaccines [76-79]. In particular, the United States Food and Drug Administration (FDA) approved three such vaccines for use in the United States, namely the Pfizer-BioNTech, Moderna and Johnson \& Johnson vaccines. Prior to the game-changing moment of the development of these vaccines, control efforts against the SARS-CoV-2 pandemic were limited to the use of non-pharmaceutical interventions, such as quarantine of suspected case, isolation of confirmed cases, use of face masks in public, social-distancing, community lockdowns, etc. Although the vaccines have proven to be very effective in significantly decreasing the burden of the SARS-CoV-2 pandemic in the United States and in other countries and/or regions with moderate to high vaccination coverage (as measured in terms of preventing new cases and minimizing severity of disease, hospitalization and death in breakthrough infections), they have not been able to lead to the elimination of the pandemic. This is largely due to the concerning level of vaccine hesitancy [21, 80-87] and emergence of numerous variants of concern [35-40, 42, 43]. The three vaccines being used in the United States were designed to target the original strain of SARS-CoV-2, and they only offer cross-protective efficacy against the variants that emerged and circulate in the population. The FDA also provided Emergency Use Authorization (EUA) for two very effective antivirals for use to treat individuals with severe symptoms of SARS-CoV-2.

Since it is now clear that vaccination alone is insufficient to lead to elimination of the pandemic, it is instructive to develop and use a mathematical modeling framework for assessing the community-wide impact of combining the vaccination program with other control strategies (NPIs, such as face mask usage, and the two antivirals) in an effort to effectively curtail the COVID-19 pandemic in the United States, and for the scenario where two variants of concern (Delta and Omicron) are co-circulating. This forms the objective of this study. We developed a mathematical model, of the form of a deterministic system of nonlinear differential equations, for assessing the combined impacts of vaccination, face mask usage and antiviral treatment on minimizing and mitigating the burden of the two COVID-19 variants. In our model formulation, vaccination is based on using either the Pfizer or Moderna vaccine (each with protective efficacy against acquisition of infection set at about 95\%). We fitted and parameterized the model using the observed daily COVID-19 case data for the United States starting from the time when Omicron first emerged (end of November 2021) to the end of January 2022. We then used the additional available data for the period from February 1, 2022 to February 22, 2022 for cross validation purpose (i.e., to validate the model). The cross-validation showed that the model perfectly predicts the case data for the time period from February 1 , 
medRxiv preprint doi: https://doi.org/10.1101/2022.02.23.22271394; this version posted February 24, 2022. The copyright holder for this preprint

(which was not certified by peer review) is the author/funder, who has granted medRxiv a license to display the preprint in perpetuity.

It is made available under a CC-BY-NC-ND 4.0 International license .

2022 to February 22, 2022.

Qualitative analysis of the model reveals that its disease-free equilibrium is locally-asymptotically stable whenever a certain epidemiological threshold, known as the control reproduction number (denoted by $\mathbb{R}_{c}$ ) is less than one. The epidemiological implication of this result is that the disease can be eliminated if the reproduction number is less than one, and will persist if it is greater than one. We computed the value of the threshold quantity $\mathbb{R}_{c}$, which is defined as the maximum of the constituent reproduction numbers for the spread of the Delta and Omicron variants, using the parametrization of the model obtained from the data fitting. This computation shows that, for the simulation period from end of November 2021 to date, while the constituent reproduction number for the Delta variant is 0.28 , that of Omicron is approximately equal to one (0.96). Thus, our study showed that Omicron is the predominant variant, and that Delta has essentially died out. Furthermore, if current baseline levels of the control measures implemented in the United States are maintained, Omicron will die out as well (but will persist, otherwise). Also, we showed that vaccine-derived herd immunity can be achieved (and the pandemic can be eliminated) if at least $68 \%$ of the population of the United States is fully-vaccinated with either the Pfizer or Moderna vaccine. Since $64 \%$ of United States population is already fully-vaccinated, our study shows that increasing the vaccination coverage in the unvaccinated population (and/or population of those who only received one vaccine dose) by about $4 \%$ could push the population to achieve herd immunity. If the level of cross-protective immunity offered by the two vaccines against the Delta and Omicron variants is lower than the baseline levels in our simulations (e.g., 60\%), the vaccination coverage needed to achieve vaccine-derived herd immunity increases (to $84 \%$ ).

We also assessed the impact of combining vaccination with mask usage using various mask types. We showed that the proportion of individuals who need to be fully-vaccinated to achieve herd immunity decreases with increasing coverage of face masks in the community (from the baseline face mask usage), and the level of reduction achieved depends on the quality of the mask used (specifically, a greater reduction in herd immunity level needed to eliminate the disease is achieved if the high-quality N95 masks are prioritized, in comparison to the scenario where moderate quality surgical masks are prioritized). We showed that greater reductions in pandemic burden are recorded using N95 masks, followed by surgical masks, and then cloth masks (as expected). Furthermore, our results show that having more people wear surgical masks is more effective than fewer people wearing N95 masks.

Although the mRNA vaccines (Pfizer, Moderna) designed to fight the original strain of SARS-CoV-2 reduce the risk of hospitalization and death and offer some cross protection against variants of concern, the cross protective efficacy they offer wane over time. In particular, the protective efficacy of these vaccines wanes down to about $40 \%$ within a few months of the second dose $[17,88,89]$. We simulated the model to assess the impact of waning vaccine-derived immunity (in fully vaccinate and boosted individuals) and natural immunity. Our simulations showed an increase in disease burden if immunity wanes at a faster rate (e.g., if both vaccine-derived and natural immunity wane within 3 months, as against the baseline of within about 9 months to a year), with the possibility of another wave of the Omicron variant (albeit a much milder one this time, with a projected peak size at least $72 \%$ lower than the Omicron peak size recorded in January of 2022). This, and the possibility of the possible future emergence of other SARS-CoV-2 variants of concern (to compete with, or displace, Omicron), suggests that a fourth Pfizer/Moderna booster dose may be needed in the United States this year (2022) to supplement the effort to eliminate the SARS-CoV-2 pandemic. For example, laboratory investigations have suggested that the BA.2 subvariant of Omicron might lead to more severe disease, and that current vaccines against COVID-19 might not be effective against this sub-variant [90-92]. In any case, our simulation results advocating for a fourth booster dose of Pfizer and Moderna for the United States, is in line with the decision in Israel to authorize a fourth booster dose against the pandemic starting with immuno-compromised individuals, adults over the age of 60 and health-care employees, and then adults aged 18 and over [93, 94]. It should be emphasized that our simulations show that only marginal increase in disease burden is observed if both the vaccine-derived and the natural immunity last at least six months.

We showed that while the use of the two approved antiviral drugs induce marginal impact in reducing the number 
medRxiv preprint doi: https://doi.org/10.1101/2022.02.23.22271394; this version posted February 24, 2022. The copyright holder for this preprint (which was not certified by peer review) is the author/funder, who has granted medRxiv a license to display the preprint in perpetuity.

It is made available under a CC-BY-NC-ND 4.0 International license .

of new daily cases of SARS-CoV-2 in the United States, their usage offer a more pronounce effect in reducing hospitalizations. Specifically, if all other interventions (vaccination and masking) are maintained at their baseline values, even the most efficient treatment strategy (e.g., one associated with treating symptomatic cases within a day or two of onset of symptoms) cannot lead to elimination of the COVID-19 pandemic until about the year 2024. However, such elimination can be achieved by increasing the effectiveness and coverages of vaccination and/or mask usage. In other words, our study showed that while treatment reduces hospitalizations, the prospect of COVID-19 elimination is enhanced by focusing investments of control resources on mask usage and vaccination (rather than on treatment options).

\section{Acknowledgments}

ABG acknowledges the support, in part, of the Simons Foundation (Award \#585022) and the National Science Foundation (Grant Number: DMS-2052363). CNN acknowledges the support of the Simons Foundation (Award \#627346).

\section{References}

[1] D. M. Morens and A. S. Fauci, "The 1918 influenza pandemic: insights for the 21st century," The Journal of infectious diseases 195, 1018-1028 (2007).

[2] I. O. Ayenigbara, O. R. Adeleke, G. O. Ayenigbara, J. S. Adegboro, and O. O. Olofintuyi, "Covid-19 (sarscov-2) pandemic: fears, facts and preventive measures," Germs 10, 218 (2020).

[3] Q. Li, X. Guan, P. Wu, X. Wang, L. Zhou, Y. Tong, R. Ren, K. S. Leung, E. H. Lau, J. Y. Wong, et al., "Early transmission dynamics in Wuhan, China, of novel coronavirus-infected pneumonia," New England Journal of Medicine (2020).

[4] C. N. Ngonghala, E. Iboi, S. Eikenberry, M. Scotch, C. R. MacIntyre, M. H. Bonds, and A. B. Gumel, "Mathematical assessment of the impact of non-pharmaceutical interventions on curtailing the 2019 novel coronavirus," Mathematical Biosciences 108364 (2020).

[5] I. F. Miller, A. D. Becker, B. T. Grenfell, and C. J. E. Metcalf, "Disease and healthcare burden of covid-19 in the united states," Nature Medicine 26, 1212-1217 (2020).

[6] Worldometer. (n.d.). , “Covid live update.” Worldometer information (Accessed on February 22, 2022). Online Version

[7] E. Dong, H. Du, and L. Gardner, "An interactive web-based dashboard to track COVID-19 in real time," The Lancet Infectious Diseases (2020).

[8] S. M. Moghadas, T. N. Vilches, K. Zhang, C. R. Wells, A. Shoukat, B. H. Singer, L. A. Meyers, K. M. Neuzil, J. M. Langley, M. C. Fitzpatrick, et al., "The impact of vaccination on coronavirus disease 2019 (covid-19) outbreaks in the united states," Clinical Infectious Diseases 73, 2257-2264 (2021).

[9] C. E. Wagner, C. M. Saad-Roy, S. E. Morris, R. E. Baker, M. J. Mina, J. Farrar, E. C. Holmes, O. G. Pybus, A. L. Graham, E. J. Emanuel, et al., "Vaccine nationalism and the dynamics and control of sars-cov-2," Science 373, eabj7364 (2021).

[10] E. Mathieu, H. Ritchie, E. Ortiz-Ospina, M. Roser, J. Hasell, C. Appel, C. Giattino, and L. Rodés-Guirao, "A global database of covid-19 vaccinations," Nature human behaviour 5, 947-953 (2021). 
medRxiv preprint doi: https://doi.org/10.1101/2022.02.23.22271394; this version posted February 24, 2022. The copyright holder for this preprint (which was not certified by peer review) is the author/funder, who has granted medRxiv a license to display the preprint in perpetuity. It is made available under a CC-BY-NC-ND 4.0 International license .

[11] Jeff Craven. (2022). , “Covid-19 vaccine tracker.” Regulator Affairs Professionals Society (RAPS). (Accessed on February 22, 2022).

Online Version

[12] Food and Drugs Administration (FDA), "FDA approves first covid-19 vaccine," FDA NEWS Release, 2021 (Accessed on February 16, 2022).

Online Version

[13] J. H. Tanne, “Covid-19: Fda approves pfizer-biontech vaccine in record time,” (2021).

[14] Centers for Disease Control and Prevention (CDC), "Different COVID-19 vaccines," CDC information (Accessed on June 25, 2021).

Online Version

[15] E. Mahase, "Covid-19: Moderna vaccine is nearly 95\% effective, trial involving high risk and elderly people shows,” BMJ: British Medical Journal (Online) 371 (2020).

[16] W. H. Self, M. W. Tenforde, J. P. Rhoads, M. Gaglani, A. A. Ginde, D. J. Douin, S. M. Olson, H. K. Talbot, J. D. Casey, N. M. Mohr, et al., "Comparative effectiveness of moderna, pfizer-biontech, and janssen (johnson \& johnson) vaccines in preventing covid-19 hospitalizations among adults without immunocompromising conditions—united states, march-august 2021," Morbidity and Mortality Weekly Report 70, 1337 (2021).

[17] N. Andrews, J. Stowe, F. Kirsebom, S. Toffa, T. Rickeard, E. Gallagher, C. Gower, M. Kall, N. Groves, A.-M. O'Connell, et al., "Effectiveness of covid-19 vaccines against the omicron (b. 1.1. 529) variant of concern," MedRxiv (2021).

[18] Centers for Disease Control and Prevention (CDC), "Cdc expands eligibility for covid-19 booster shots to all adults," CDC information (Accessed on February 22, 2022).

Online Version

[19] H. S. Sacks, "The single-dose j\&j vaccine had $67 \%$ efficacy against moderate to severe-critical covid-19 at $\geq$ 14 d," Annals of Internal Medicine 174, JC75 (2021).

[20] Centers for Disease Control and Prevention, "Different COVID-19 vaccines," CDC information (Accessed on January 25, 2021).

Online Version

[21] V. C. Lucia, A. Kelekar, and N. M. Afonso, "Covid-19 vaccine hesitancy among medical students," Journal of Public Health 43, 445-449 (2021).

[22] L. J. Abu-Raddad, H. Chemaitelly, and A. A. Butt, "Effectiveness of the bnt162b2 covid-19 vaccine against the b. 1.1. 7 and b. 1.351 variants," New England Journal of Medicine 385, 187-189 (2021).

[23] T. Koyama, D. Weeraratne, J. L. Snowdon, and L. Parida, "Emergence of drift variants that may affect covid19 vaccine development and antibody treatment," Pathogens 9, 324 (2020).

[24] J. S. Tregoning, K. E. Flight, S. L. Higham, Z. Wang, and B. F. Pierce, "Progress of the covid-19 vaccine effort: viruses, vaccines and variants versus efficacy, effectiveness and escape," Nature Reviews Immunology 21, 626-636 (2021).

[25] S. Loomba, A. de Figueiredo, S. J. Piatek, K. de Graaf, and H. J. Larson, "Measuring the impact of covid-19 vaccine misinformation on vaccination intent in the uk and usa," Nature human behaviour 5, 337-348 (2021).

[26] E. Dubé, C. Laberge, M. Guay, P. Bramadat, R. Roy, and J. A. Bettinger, "Vaccine hesitancy: an overview," Human vaccines \& immunotherapeutics 9, 1763-1773 (2013). 
medRxiv preprint doi: https://doi.org/10.1101/2022.02.23.22271394; this version posted February 24, 2022. The copyright holder for this preprint (which was not certified by peer review) is the author/funder, who has granted medRxiv a license to display the preprint in perpetuity. It is made available under a CC-BY-NC-ND 4.0 International license .

[27] C. Jarrett, R. Wilson, M. O’Leary, E. Eckersberger, H. J. Larson, et al., "Strategies for addressing vaccine hesitancy-a systematic review," Vaccine 33, 4180-4190 (2015).

[28] P. Paterson, F. Meurice, L. R. Stanberry, S. Glismann, S. L. Rosenthal, and H. J. Larson, "Vaccine hesitancy and healthcare providers," Vaccine 34, 6700-6706 (2016).

[29] M. Siddiqui, D. A. Salmon, and S. B. Omer, "Epidemiology of vaccine hesitancy in the united states," Human vaccines \& immunotherapeutics 9, 2643-2648 (2013).

[30] N. E. MacDonald et al., "Vaccine hesitancy: Definition, scope and determinants," Vaccine 33, 4161-4164 (2015).

[31] The U.S. Food and Drug Administration(FDA), "Coronavirus (covid-19) update: Fda authorizes additional oral antiviral for treatment of covid-19 in certain adults," FDA NEWS RELEASE (Accessed on February 24, 2022).

Online Version

[32] Pfizer Inc. (NYSE: PFE).(2022)., "Pfizer shares in vitro efficacy of novel covid-19 oral treatment against omicron variant," Pfizer information (Accessed on February 24, 2022).

Online Version

[33] A. Jayk Bernal, M. M. Gomes da Silva, D. B. Musungaie, E. Kovalchuk, A. Gonzalez, V. Delos Reyes, A. Martín-Quirós, Y. Caraco, A. Williams-Diaz, M. L. Brown, et al., "Molnupiravir for oral treatment of covid-19 in nonhospitalized patients," New England Journal of Medicine (2021).

[34] Food and Drugs Administration (FDA), "Coronavirus (COVID-19) update: FDA authorizes first oral antiviral for treatment of COVID-19," FDA NEWS Release, 2021 (Accessed on February 16, 2022).

Online Version

[35] E. Mahase, “Covid-19: What new variants are emerging and how are they being investigated?” (2021).

[36] A. Gómez-Carballa, J. Pardo-Seco, X. Bello, F. Martinón-Torres, and A. Salas, "Superspreading in the emergence of covid-19 variants," Trends in Genetics 37, 1069-1080 (2021).

[37] Centers for Disease Control and Prevention (CDC), "Variant proportions," CDC information (Accessed on February 24, 2022).

Online Version

[38] S. S. A. Karim and Q. A. Karim, "Omicron sars-cov-2 variant: a new chapter in the covid-19 pandemic," The Lancet 398, 2126-2128 (2021).

[39] D. Duong, "What's important to know about the new covid-19 variants?" (2021).

[40] D. Geers, M. C. Shamier, S. Bogers, G. den Hartog, L. Gommers, N. N. Nieuwkoop, K. S. Schmitz, L. C. Rijsbergen, J. A. van Osch, E. Dijkhuizen, et al., "Sars-cov-2 variants of concern partially escape humoral but not t cell responses in covid-19 convalescent donors and vaccine recipients," Science immunology 6, eabj1750 (2021).

[41] P. Mlcochova, S. A. Kemp, M. S. Dhar, G. Papa, B. Meng, I. A. Ferreira, R. Datir, D. A. Collier, A. Albecka, S. Singh, et al., "Sars-cov-2 b. 1.617. 2 delta variant replication and immune evasion," Nature 599, 114-119 (2021).

[42] C. Del Rio, S. B. Omer, and P. N. Malani, "Winter of omicron-the evolving covid-19 pandemic," JAMA (2021). 
medRxiv preprint doi: https://doi.org/10.1101/2022.02.23.22271394; this version posted February 24, 2022. The copyright holder for this preprint (which was not certified by peer review) is the author/funder, who has granted medRxiv a license to display the preprint in perpetuity. It is made available under a CC-BY-NC-ND 4.0 International license .

[43] E. Callaway, H. Ledford, et al., "How bad is Omicron? what scientists know so far," Nature 600, 197-199 (2021).

[44] E. Dong, H. Du, and L. Gardner, “Coronavirus COVID-19 Global Cases by Johns Hopkins CSSE," The Lancet Infectious Diseases (2020).

Online Version

[45] B. J. Gardner and A. M. Kilpatrick, "Estimates of reduced vaccine effectiveness against hospitalization, infection, transmission and symptomatic disease of a new sars-cov-2 variant, omicron (b. 1.1. 529), using neutralizing antibody titers," MedRxiv (2021).

[46] M. Betti, N. Bragazzi, J. Heffernan, J. Kong, and A. Raad, "Could a new covid-19 mutant strain undermine vaccination efforts? a mathematical modelling approach for estimating the spread of b. 1.1. 7 using ontario, canada, as a case study," Vaccines 9, 592 (2021).

[47] G. Gonzalez-Parra, D. Martínez-Rodríguez, and R. J. Villanueva-Micó, "Impact of a new sars-cov-2 variant on the population: A mathematical modeling approach," Mathematical and Computational Applications 26, 25 (2021).

[48] M. Mancuso, S. E. Eikenberry, and A. B. Gumel, "Will vaccine-derived protective immunity curtail covid-19 variants in the us?" Infectious Disease Modelling 6, 1110-1134 (2021).

[49] O. Alagoz, A. K. Sethi, B. W. Patterson, M. Churpek, G. Alhanaee, E. Scaria, and N. Safdar, "The impact of vaccination to control covid-19 burden in the united states: A simulation modeling approach," PloS one 16, e0254456 (2021).

[50] A. Shoukat, T. N. Vilches, S. M. Moghadas, P. Sah, E. C. Schneider, J. Shaff, A. Ternier, D. A. Chokshi, and A. P. Galvani, "Lives saved and hospitalizations averted by covid-19 vaccination in new york city: a modeling study," The Lancet Regional Health-Americas 5, 100085 (2022).

[51] J. Roy, S. Heath, D. Ramkrishna, and S. Wang, "Modeling of covid-19 transmission dynamics on us population: Inter-transfer infection in age groups, mutant variants, and vaccination strategies," medRxiv (2021).

[52] A. ul Rehman, R. Singh, and P. Agarwal, "Modeling, analysis and prediction of new variants of covid-19 and dengue co-infection on complex network," Chaos, Solitons \& Fractals 150, 111008 (2021).

[53] A. Ramos, M. Vela-Pérez, M. Ferrández, A. Kubik, and B. Ivorra, "Modeling the impact of sars-cov-2 variants and vaccines on the spread of covid-19," Communications in Nonlinear Science and Numerical Simulation 102, 105937 (2021).

[54] A. B. Gumel, E. A. Iboi, C. N. Ngonghala, and G. A. Ngwa, "Toward achieving a vaccine-derived herd immunity threshold for covid-19 in the us," Frontiers in Public Health 9 (2021).

[55] L. Matrajt, E. R. Brown, D. Dimitrov, and H. Janes, "The role of antiviral treatment in curbing the covid-19 pandemic: a modeling study," Medrxiv (2021).

[56] H. T. Banks, M. Davidian, J. R. Samuels, and K. L. Sutton, An Inverse Problem Statistical Methodology Summary, 249-302 (Springer Netherlands, Dordrecht, 2009).

Online Version

[57] G. Chowell, "Fitting dynamic models to epidemic outbreaks with quantified uncertainty: a primer for parameter uncertainty, identifiability, and forecasts," Infectious Disease Modelling 2, 379-398 (2017).

[58] C. N. Ngonghala, E. Iboi, S. Eikenberry, M. Scotch, C. R. MacIntyre, M. H. Bonds, and A. B. Gumel, "Mathematical assessment of the impact of non-pharmaceutical interventions on curtailing the 2019 novel coronavirus," Mathematical Biosciences. 325, 108364 (2020). 
medRxiv preprint doi: https://doi.org/10.1101/2022.02.23.22271394; this version posted February 24, 2022. The copyright holder for this preprint (which was not certified by peer review) is the author/funder, who has granted medRxiv a license to display the preprint in perpetuity.

It is made available under a CC-BY-NC-ND 4.0 International license .

[59] A. A. King, M. Domenech de Cellès, F. M. Magpantay, and P. Rohani, "Avoidable errors in the modelling of outbreaks of emerging pathogens, with special reference to ebola," Proceedings of the Royal Society B: Biological Sciences 282, 20150347 (2015).

[60] O. Diekmann, J. A. P. Heesterbeek, and J. A. Metz, "On the definition and the computation of the basic reproduction ratio $R_{0}$ in models for infectious diseases in heterogeneous populations," Journal of Mathematical Biology 28, 365-382 (1990).

[61] P. van den Driessche and J. Watmough, "Reproduction numbers and sub-threshold endemic equilibria for compartmental models of disease transmission," Mathematical Biosciences 180, 29-48 (2002).

[62] E. A. Iboi, C. N. Ngonghala, and A. B. Gumel, "Will an imperfect vaccine curtail the COVID-19 pandemic in the US?” Infectious Disease Modelling 5, 510-524 (2020).

[63] A. B. Gumel, E. A. Iboi, C. N. Ngonghala, and E. H. Elbasha, "A primer on using mathematics to understand covid-19 dynamics: Modeling, analysis and simulations," Infectious Disease Modelling 6, 148-168 (2021).

[64] US Coronavirus vaccine tracker, "What's the nation's progress on vaccinations?" USA facts (Accessed on February 16, 2022).

Online Version

[65] Centers for Disease Control and Prevention (CDC), "COVID-19 vaccinations in the United States," CDC COVID-19 data tracker (Accessed on February 16, 2022).

Online Version

[66] S. E. Eikenberry, M. Muncuso, E. Iboi, T. Phan, E. Kostelich, Y. Kuang, and A. B. Gumel, "To mask or not to mask: Modeling the potential for face mask use by the general public to curtail the COVID-19 pandemic," Infectious Disease Modeling 5, 293-308 (2020).

[67] P. Hodjat, P. A. Christensen, S. Subedi, D. W. Bernard, R. J. Olsen, and S. W. Long, "The reemergence of seasonal respiratory viruses in Houston, Texas, after relaxing COVID-19 restrictions," Microbiology Spectrum 9, e00430-21 (2021).

[68] G. P. Guy, G. M. Massetti, and E. Sauber-Schatz, "Mask mandates, on-premises dining, and COVID-19," JAMA 325, 2199-2200 (2021).

[69] The New York Times, "The U.S. states that are ending mask mandates," The New York Times (Accessed on February 19, 2022).

Online Version

[70] Y. Avila, B. Harvey, J. C. Lee, and J. W. Shaver, "See mask mandates and guidance in each state," The New York Times (Accessed on February 19, 2022).

Online Version

[71] M. Hersher, "Nearly half of state mask mandates have ended in the past 3 weeks," NBC News (Accessed on February 19, 2022).

Online Version

[72] NIOSH, "42 CFR 84 respiratory protective devices; final rules and notice. US Centers for Disease Control and Prevention, National Institute for Occupational Safety and Health,” Federal Register 60, 110 (1997).

[73] N. H. Leung, D. K. Chu, E. Y. Shiu, K.-H. Chan, J. J. McDevitt, B. J. Hau, H.-L. Yen, Y. Li, D. K. Ip, J. Peiris, et al., "Respiratory virus shedding in exhaled breath and efficacy of face masks," Nature medicine 26, 676-680 (2020). 
medRxiv preprint doi: https://doi.org/10.1101/2022.02.23.22271394; this version posted February 24, 2022. The copyright holder for this preprint (which was not certified by peer review) is the author/funder, who has granted medRxiv a license to display the preprint in perpetuity. It is made available under a CC-BY-NC-ND 4.0 International license .

[74] Centers for Disease Control and Prevention, "NIOSH-approved particulate filtering facepiece respirators," The National Personal Protective Technology Laboratory (NPPTL) (Accessed on July 24, 2021). Online Version

[75] W. Lindsley, F. Blachere, B. Law, D. Beezhold, and D. John, "Efficacy of face masks, neck gaiters and face shields for reducing the expulsion of simulated cough-generated aerosols," Aerosol Science and Technology 55 (2021).

[76] N. Lurie, M. Saville, R. Hatchett, and J. Halton, "Developing covid-19 vaccines at pandemic speed," New England Journal of Medicine 382, 1969-1973 (2020).

[77] B. S. Graham, “Rapid covid-19 vaccine development,” Science 368, 945-946 (2020).

[78] S. Su, L. Du, and S. Jiang, "Learning from the past: development of safe and effective covid-19 vaccines," Nature Reviews Microbiology 19, 211-219 (2021).

[79] J. L. Bernal, N. Andrews, C. Gower, E. Gallagher, R. Simmons, S. Thelwall, J. Stowe, E. Tessier, N. Groves, G. Dabrera, et al., "Effectiveness of covid-19 vaccines against the b. 1.617. 2 (delta) variant," New England Journal of Medicine (2021).

[80] G. Troiano and A. Nardi, "Vaccine hesitancy in the era of covid-19," Public health 194, 245-251 (2021).

[81] S. Machingaidze and C. S. Wiysonge, "Understanding covid-19 vaccine hesitancy," Nature Medicine 27, 1338-1339 (2021).

[82] A. A. Dror, N. Eisenbach, S. Taiber, N. G. Morozov, M. Mizrachi, A. Zigron, S. Srouji, and E. Sela, "Vaccine hesitancy: the next challenge in the fight against COVID-19," European journal of epidemiology 35, 775-779 (2020).

[83] P. Soares, J. V. Rocha, M. Moniz, A. Gama, P. A. Laires, A. R. Pedro, S. Dias, A. Leite, and C. Nunes, "Factors associated with covid-19 vaccine hesitancy," Vaccines 9, 300 (2021).

[84] A. Coustasse, C. Kimble, and K. Maxik, "Covid-19 and vaccine hesitancy: a challenge the united states must overcome," The Journal of ambulatory care management 44, 71-75 (2021).

[85] A. Fridman, R. Gershon, and A. Gneezy, "Covid-19 and vaccine hesitancy: A longitudinal study," PloS one 16, e0250123 (2021).

[86] J. Murphy, F. Vallières, R. P. Bentall, M. Shevlin, O. McBride, T. K. Hartman, R. McKay, K. Bennett, L. Mason, J. Gibson-Miller, et al., "Psychological characteristics associated with covid-19 vaccine hesitancy and resistance in ireland and the united kingdom," Nature communications 12, 1-15 (2021).

[87] M. Sallam, "Covid-19 vaccine hesitancy worldwide: a concise systematic review of vaccine acceptance rates," Vaccines 9, 160 (2021).

[88] H. F. Tseng, B. K. Ackerson, Y. Luo, L. S. Sy, C. Talarico, Y. Tian, K. Bruxvoort, J. E. Tupert, A. Florea, J. H. $\mathrm{Ku}$, et al., "Effectiveness of mrna-1273 against sars-cov-2 omicron and delta variants," medRxiv (2022).

[89] C. Willyard et al., "What the omicron wave is revealing about human immunity," Nature 602, 22-25 (2022).

[90] B. Goodman, “As BA.2 subvariant of Omicron rises, lab studies point to signs of severity," CNN (Accessed on February 21, 2022).

Online Version

[91] D. Yamasoba, I. Kimura, H. Nasser, Y. Morioka, N. Nao, J. Ito, K. Uriu, M. Tsuda, J. Zahradnik, K. Shirakawa, et al., "Virological characteristics of SARS-CoV-2 BA.2 variant," bioRxiv (2022). 
medRxiv preprint doi: https://doi.org/10.1101/2022.02.23.22271394; this version posted February 24, 2022. The copyright holder for this preprint (which was not certified by peer review) is the author/funder, who has granted medRxiv a license to display the preprint in perpetuity. It is made available under a CC-BY-NC-ND 4.0 International license .

[92] J. Chen and G.-W. Wei, "Omicron BA. 2 (B. 1.1. 529.2): high potential to becoming the next dominating variant,” arXiv preprint arXiv:2202.05031 (2022).

[93] D. Williams, "Israel mulls offering 4th COVID vaccine dose to all adults," Reuters (Accessed on February 21, 2022).

Online Version

[94] T. K. Burki, "Fourth dose of COVID-19 vaccines in Israel," The Lancet Respiratory Medicine 10, e19 (2022). Online Version 